\title{
A faunistic review of ground beetles of Lebiinae Bonelli, 1810 (Coleoptera: Carabidae) of Lithuania
}

\author{
Vytautas Tamutis \& Arvids Barševskis
}

Tamutis, V. \& Barševskis, A. 2014: A faunistic review of ground beetles of Lebiinae Bonelli, 1810 (Coleoptera: Carabidae) of Lithuania. — Entomol. Fennica 25: $65-85$.

The paper presents the first review on species composition and distribution of ground beetles of Lebiinae Bonelli, 1810, subfamily (Coleoptera: Carabidae) in Lithuania. During the study, all available related published sources have been reviewed. A total of 482 records have been analyzed and more than 700 specimens collected since 1923 have been examined. Lebiinae subfamily is presented by 2 tribes in Lithuania. The tribe Odocanthini is monotypical with a single species. The other one, Lebiini tribe, is more diverse, represented by 4 subtribes, 7 genera and 24 species. The information on distribution, frequency and some features of ecology and zoogeography of 25 Lebiinae species found in Lithuanian is detailed. Demetrias atricapillus (Linnaeus, 1758) is reported for the first time for Lithuanian fauna and Philorhizus notatus (Stephens, 1827) is removed from the list of Lithuanian beetles.

V. Tamutis, Kaunas T. Ivanauskas Zoological Museum, Laisvès al. 106, LT44253 Kaunas, Lithuania, and Institute of Biology and Plant Biotechnology, Aleksandras Stlginskis University, Studentu 11, Akademija, Kaunas distr., LT53361,Lithuania; E-mail: dromius@yahoo.com

A. Barševskis, Institute of Systematic Biology, Daugavpils University, Vienibas iela 13, Daugavpils, LV-5400,Latvia; E-mail: arvids.barsevskis@du.lv

Received 29 April 2013, accepted 1 October 2013

\section{Introduction}

The ground beetles of the Lebiinae subfamily are characterized by a transversally or obliquely truncated elytron, without a membranous fringe at the apex, and which does not cover the entire abdomen. The front tibia has a smooth outer margin.

This subfamily is a very diverse group worldwide, containing about 4,000 species and 200 genera, mainly occurring in the tropics and subtropics. Only 237 species and subspecies belonging to 27 genera occur in Europe (Kabak 2003).
Most species are found in the southern part of Europe and only 33 species belonging to 11 genera occur in northern countries, such as Norway, Sweden, Denmark, Finland, Russia (northern and central parts of European Russia), Estonia, Latvia, Belarus, Poland (northern and central parts) and Lithuania (Burakowski et al. 1974, Krizhanovskij et al. 1995, Silfverberg 2010).

The first data on Lithuanian Lebiinae species was reported by Ogijewicz in 1933, presenting data on eight species found in Vilnius and Trakai environs. The list of this group of Lithuanian carabids was suplemented later by other scientists 


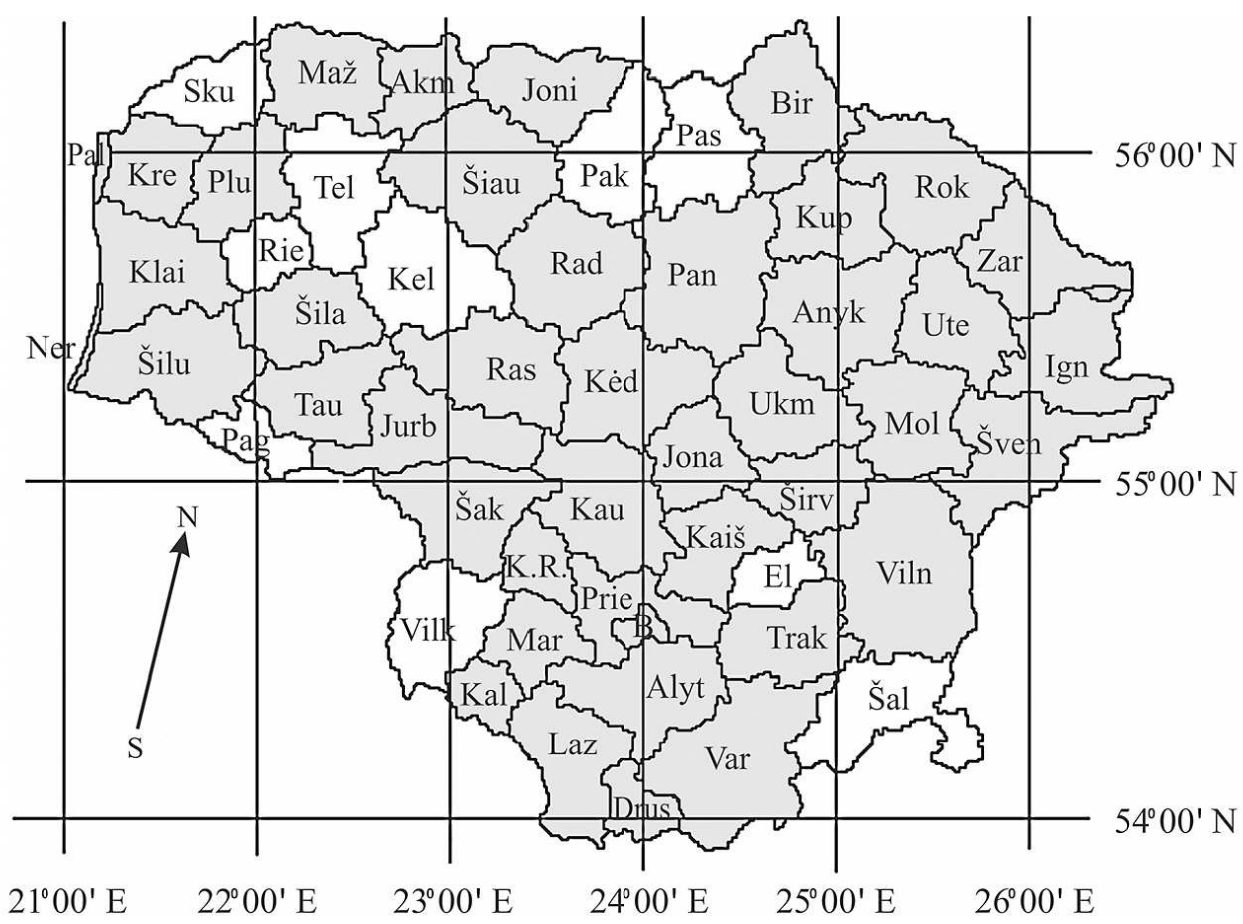

Fig. 1. Map of Lithuania with administrative districts according to a system used since 2000 . Districts where material was collected are shaded:

$A k m$ - Akmenè, Alyt - Alytus, Anyk - Anykščiai, Birž - Biržai, B.- Birštonas, El - Elektrènai, Drus Druskininkai, Ign - Ignalina, Jona- Jonava, Joni - Joniškis, Jurb - Jurbarkas, Kaiš - Kašiadorys, Kau Kaunas, Kel-Kelmè, Kèd - Kèdainiai, Klai - Klaipèda, Kre - Kretinga, Kup - Kupiškis, K.R. - Kazlų Rūda, Laz - Lazdijai, Mar - Marijampolè, Maž - Mažeikiai, Mol - Molètai, Ner - Neringa, Pag Pagègiai, Pak - Pakruojis, Pal - Palanga, Pan - Panevėžys, Pas - Pasvalys, Plu - Plungè, Prie Prienai, Rad - Radviliškis, Ras - Raseiniai, Rie - Rietavas, Rok - Rokiškis, Sku - Skuodas, Šak Šakiai, Šal - Šalčininkai, Šiau - Šiauliai, Šila - Šilalè, Šilu - Šilutè, Širv-Širvintos, Šven- Švenčionys, Tau - Tauragè, Tel - Telšiai, Trak - Trakai, Ukm - Ukmergè, Ut - Utena, Var - Varèna, Vilk Vilkaviškis, Viln - Vilnius, Zar - Zarasai.

(Pileckis 1960, 1976, Sharova \& Grüntal 1973, Pileckis \& Monsevičius 1982, 1995, Miländer et al. 1984, Barševskis 2001, Ferenca et al. 2002, Tamutis et al. 2008). Some information on the species of Lebiinae of Lithuania is presented in some well-known catalogues or lists of the beetles of related territories (Silfverberg 1992, 2004, 2010, Krizhanovskij et al. 1995, Kabak 2003), for example. The most comprehensive list of Lithuanian carabids is presented in a recently published catalogue of Lithuanian beetles (Tamutis et al. 2011). Therein are listed 25 species belonging to 12 genera of Lebiinae, and eight other species expected to be found in Lithuania are noted. The name of Lamprias Bonelli, 1810 is contained therein and is considered as a valid genus name. However, the species composition of
Lithuanian Lebiinae is still not completely disclosed.

Compared to other ground beetles living in Lithuania, Lebiinae are characterized by their diverse biology. The ground beetles in general are known as typical ground dwellers, but for example Odacantha, Lebia and Demetrias species are perfect grass climbers. Furthermore, all Dromius and Calodromius species are exclusively tree habitants. The larvae of Lebia species are distinguished by their very specific life cycle and food specialization. The ground dwelling Paradromius, Microlestes, Syntomus, Lionychus and $\mathrm{Cy}$ mindis species are mostly xerophilous, occurring only in dry, open areas.

Due to the specificity of their lifestyle, the ecological, faunistic and other biological investi- 
gations of these ground beetles are quite problematic. For this reason, both the data on their prevalence and bionomics is still incomplete. This is especially so in Lithuania, where no directed systematic investigations have been done. Thus, all Lebiinae species are considered as rare or very rare and have not been discovered in large numbers in Lithuania (Pileckis \& Monsevičius 1995). Such circumstances were the main reason to compile a comprehensive faunistic review of Lithuanian Lebiinae ground beetles. Our study was based on analysis of all related published sources, examination of all available specimens of Lithuanian Lebiinae, as well as on our own observed data and collected material.

\section{Material and methods}

All existing Lithuanian records of Lebiinae ground beetles are included in this review. The specimens collected by Auksé Abromaitytè (A. A.), Algimantas Jakimavičius (A. J.), Arvids Barševskis (A. B.), Katrīna Barševska (K. B.), Bronius Jakaitis (B. J.), A. Kaulinis (A. K.), Aleksandras Meržijevskis (A. M.), A. Šumskaite (A. Š.), Borys Ogijewicz (B. O.), Bronius Šablevičius (B. Š.), Brigita Tamute (B. T.), Dalius Dapkus (D. D.), Elena Gaidienè (E. G.), Artūras Gedminas (A. G.), Giedrius Švitra (G. Š.), Henrikas Ostrauskas (H. O.), Kęstutis Arbačiauskas (K. A.), Kalle Lundsten (K. L.), Linas Tiškevičius (L. T.), Norbertas Noreika (N. N.), Povilas Ivinskis (P. I.), Paulius Plastininas (P. P.), Paulius Zolubas (P. Z.), Rolandas Dvilevičius (R. D.), Romas Ferenca (R. F.), Rimantas Pankevičius (R. P.), Edgars Rudāns (E. R.), Saulius Gaižutis (S. G.), Saulius Karalius (S. K.), Saulius Pakalniškis (S. Pa.), Simonas Pileckis (S. Pi.), Vitolis Gavelis (V. G.), Vytautas Inokaitis (V. I.), Vidmantas Monsevičius (V. M.), Vytautas Jonaitis (V. J.) and Vytautas Tamutis (V. T.) in the period from 1923 to 2013 were examined or reexamined in this study.

During the last 30 years, special attention to the studies of Lebiinae ground beetles has been paid in Lithuania. More than $77 \%$ of all examined material has been collected during this period. More intensive studies have been done by
Vytautas Tamutis, Romas Ferenca, Povilas Ivinskis and Vidmantas Monsevičius in few regions during the mentioned period: Baltic Sea coast region and Akmenè, Šiauliai, Kaunas and Marijampole districts (Fig. 1).

The methods for collecting the material were chosen so that information on the specific ecological aspects of the beetles could be obtained. Visual searching of soil surface, under the moss, plant debris, bark chips, barks and stem surface was most commonly used. Some species have been caught also by using the sweep net as well as pitfall and barrier (window) traps, or by shaking and beating from reeds, trees and bushes. Our material was collected from 42 districts ( $80 \%$ of all districts) of Lithuania (Fig. 1.).

The examined material is deposited in following Lithuanian and foreign collections:

- Kaunas T. Ivanauskas Zoological Museum (Kaunas)

- Vilnius University (Vilnius)

- Nature Research Centre, Institute of Ecology (Vilnius)

- Private collection of Aleksandras Meržijevskis (Kaunas)

- Daugavpils University, Institute of Systematic Biology, Coleopterological Research Centre (Ilgas, Latvia)

The classification of ground beetles follows that suggested by Anichtchenko et al. (2007-2012).

The identification keys suggested by Freude (1976), Sciaky (1991), Hurka (1996) and Sundukov (2004) were used.

The temporal distribution of the species is based on the summary of all records.

Explanations of the abbreviations used: env.environs; f. - forest; t. - town, Nat. - Nature, Land. - Landscape, Ent. - Entomological, Orn. Ornitological, B.-Z. - Botanical-Zoological, R. Reserve.

\section{Review of the material}

During the study, a total of 482 records have been analysed and more than 700 specimens have been examined. 


\subsection{Tribe Lebiini Bonelli, 1810}

\subsubsection{Subtribe Cymindiina Laporte, 1834}

Genus Cymindis Latreille, 1806

Subgenus Cymindis Latreille, 1806

C. (C.) angularis Gyllenhal, 1810

Examined material. Klai: Karklė env., 07.IX. 2005, 18.X.2005 (2, 3, caught by pitfall traps, leg. P. I.); Ner: Smiltyné env., 04.V.1989, 03.V.2000 (1, 1, leg. S. K.), Alksnynė env., 30.VIII.-13.IX. 2010 (1, open place after forest fire, caught by pitfall trap, leg. P. I.).

Earlier records. Only one record older than 70 years is known, from Varéna district (leg. A. Palionis) (Ferenca 2006).

General distribution. Europe, Kazakhstan, Kirgystan, southern parts of West and Middle Siberia. Not indicated for Lithuania in Catalogue of Palaearctic Coleoptera (Kabak 2003).

Notes. The species is xerophilic, occurring mainly in open, dry, sun-exposed habitats with sand or gravel and sparse vegetation. It is insufficiently known in Lithuania. Recent findings are located only in dune regions of the Baltic Sea.

\section{C. (C.) humeralis (Geoffroy, 1875)}

Examined material. Not confirmed by authors.

Earlier records. Vilnius district (Miländer et al. 1984).

General distribution. Europe, to the north as far as southern Sweden, Lithuania?, Belarus. Not indicated for Lithuania in Catalogue of Palaearctic Coleoptera (Kabak 2003).

Notes. The species is xerophilic, occurring mainly in open, dry, sun-exposed habitats with sand or gravel and sparse vegetation. It is very rare throughout the northern border of its distribution range.

Subgenus Pseudomastinus Bousquet, 2002

$C$. $(P$.) macularis Mannerheim in Fischer von Waldheim, 1824

Examined material. Var: Varèna env., 01.VII. 1966 (1, leg. S. Pi.), Čepkeliai Nat. R., 29.VII. 1976 (1, leg. V. M.)

Earlier records. The findings are single re- cords of the species in Lithuania. The latter one above was the source for inclusion of this species to the list of beetles of Cepkeliai Nature Reserve (Monsevičius 1997). The notification of this species in Lithuanian Baltic Sea region (Pileckis \& Monsevičius 1995) is not confirmed.

General distribution. Europe, Kazakhstan, southern parts of West and Middle Siberia. Not indicated for Lithuania in Catalogue of Palaearctic Coleoptera (Kabak 2003).

Notes. The species is xerophilic, inhabiting sandy and very dry areas with sparse vegetation. It is insufficiently known in Lithuania, only from southern part of the country.

C. (P.) vaporariorum (Linnaeus, 1758)

Examined material. Jurb: Liepgiriai f., 22.VI. 1971 (1, leg. B. J.); Kau: Dubrava f., 12.VII. 1984 (1, leg. R. F.), 27.IX.2007, 23.V.2008 (1, 1, leg. A. G.), Meištiné f., 28.VI.2006 (1, leg. A. M.); Šak: Juškinè f., 05.VII.2009 (1, leg. R. F.); Šilu: Aukštumala peatbog, June-October 2002 (3, pitfall traps, leg. R. F.); Šven: Baluoša peatbog, 15.VI.1999 (1, pitfall trap, leg. D. D.); Viln: Kregžlè f., 11.VII.2005, 02.VIII.2005 (1, 2, pitfall traps, leg. P. I.).

Earlier records. Previous exact records on the distribution of this species in Lithuania are missing. It was noted for Juodkrante (Schwarzort) environs (Bercio \& Folwaczny 1979) and NorthWestern Region of Lithuania (Pileckis \& Monsevičius 1995) without definite data.

General distribution. Europe (only in mountains in southern regions of Europe), Kazakhstan, southern part of Siberia, Mongolia, northern China, Far East, Japan. Not indicated for Lithuania in Catalogue of Palaearctic Coleoptera (Kabak 2003).

Notes. As the other species of the genus, this is a xerophilic species. However, it tolerates less dry habitats, such as the moderate shade of sparse pines or forest edges, as well as dry peat soils. Vegetation must be low and sparse, consisting of Calluna, Empetrum, Arctostaphylos, Vaccinium vitis-idaea, etc. It is the commonest species of the genus in Lithuania, but the distribution is sporadic. All found specimens were collected on dry sandy or peaty heaths. 


\subsubsection{Subtribe Demetriadina Bates, 1886}

Genus Demetrias Bonelli, 1810

Subgenus Aetophorus Schmidt-Göbel, 1846

D. (Ae.) imperialis (Germar, 1824)

Examined material. Kaǐs: Bundiškès env., 21.VIII.2002 (1, wet meadow near reeds' overgrowth, leg. V. T.); Kup: Palevenėlé env., 12.IX. 1999 (2, leg. R. P.); Ner: Juodkrante env., 02.VII. 2005 (1, leg. R. F.); Pervalka env., 20.V.2011 (1, leg. R. F.), Juodkranté env., 06.VII.2012 (2, on the stems of reeds, leg. V. T.); Silu: Ventes Ragas env., 26.VIII.1988 (1, leg. R. F.); Sirv: Antanèliai env., 06.V.2012 (2, reed bed, leg. V. T.).

Earlier records. Only two records known before in Lithuania, in Zarasai and Neringa districts (Barševskis 2001, Ferenca 2004).

General distribution. Europe, Northern Africa, Asia Minor, Kazakhstan, Kirgyzstan. Not indicated for Lithuania in Catalogue of Palaearctic Coleoptera (Kabak 2003).

Notes. This species inhabits reed stands of swamps and standing waters and seems to be mostly restricted to live on the reeds and to avoid running on the soil surface (Schürstedt et al. 2000). We observed this species predominantly in large reed stands or their surroundings, but the occurrence everywhere was sporadic.

Subgenus Demetrias Bonelli, 1810

D. (D.) atricapillus (Linnaeus, 1758)

Examined material. Kau: Noreikiškès env., (545'05'N, 2350'08’'E) 23.V.1996 (1, littoral of a pond, leg. V. T.).

Earlier records. None.

General distribution. Europe, Northern Africa, Asia Minor, Near East, Caucasus.

Notes. This species is common in Western Europe and it is known as an active aphid predator in cereals (Lövei et al. 2003), but it is sparse or very rare in eastern countries (Burakowski et al. 1974, Barševskis 2003, Alekseev 2008). It occurs in unshaded habitats: shrubby floodplains, fields and grasslands. This is a new species for Lithuania. This record was erroneously related to Paradromius longiceps Dejean (Tamutis et al. 2008).
D. (D.) monostigma Samouelle, 1819

Examined material. Kau: Novaraistis Orn. R., 17.IV.2011 (1, under plant debris, leg. V. T.); Braziūkai env., 16.IV.2012 (1, under plant debris, leg. V. T.), 25.III.2012 (2, under plant debris, leg. V. T.), 02.IX.2012 (4, Calamagrostis epigejos overgrowth, leg V. T.); Kèd: Vincentavas env., 15.V. 2013 (1, moist meadow, leg. V. T.); Kre: Monciškè env., 09.V.2001 (1, leg. R. F.), 14.VI.2001 (3, leg. R. F.); Kup: Vasniūnai šilas f., 13.VI.1997 (1, leg. R. P.); Antašava env., 12.V.1995 (2, leg. R. P.); Mar: Riečiai env., Žuvintas Nat. R., 26.V.1981 (1, leg. V. J.); Ner: Smiltynė env., 03.V.2000, 05.VI.2000 (1, 3, leg. S. K.), Juodkrantė env., 21.VI.2007 (1, leg. R. F.), Juodkrantė env., 12.IX.2008 (1, wet forest with Alnus glutinosa, leg. V. T.), 05.X.2010 (6, dunes, C. epigejos and Ammophila arenaria overgrowth, leg. V. T.), 26.X.2010 (1, leg. R. F.); Šiau: Slydžiai env., 10.VIII.2002, 04.V.2013 (1, reed bed, leg. V. T.); Šilu: Ventès Ragas env., 31.V.1988 (1, leg. R. F.); Tau: Viešvilè Nat. R., Glitis bog, 28.VII.2008 (1, leg. V. T.); Var: Čepkeliai Nat. R., Pogarenda env., 18.IV.1981 (1, leg. V. M.).

Earlier records. Several earlier finding places have been known from Curronian Spit and Baltic sea coast regions (Ferenca et al. 2002, 2006, Šablevičius 2003) and Viešvilè Nature Reserve (Ferenca 2004). It has been also noted for Žuvintas and Čepkeliai Nature Reserves without definite data (Monsevičius 1997).

General distribution. Europe, Caucasus, Kazakhstan, Kirgyzstan.

Notes. Contrary to $D$. imperialis, this species can dwell both in wet habitats (reed beds, swamps) and xerophilic biotopes (heathlands, sand dunes) (Wachman et al. 1995, Desender 1996, Telfer \& Eversham 1996). It is not rare in Lithuania, plenty of adults can be observed climbing on reed stems from May to June (Fig. 2a) and hibernating in dry places in the grass humps.

\subsubsection{Subtribe Dromiina Bonelli, 1810}

Genus Calodromius Reitter, 1905

C. spilotus (Illiger, 1798)

Examined material. Akm: Kamanos Nat. R., 

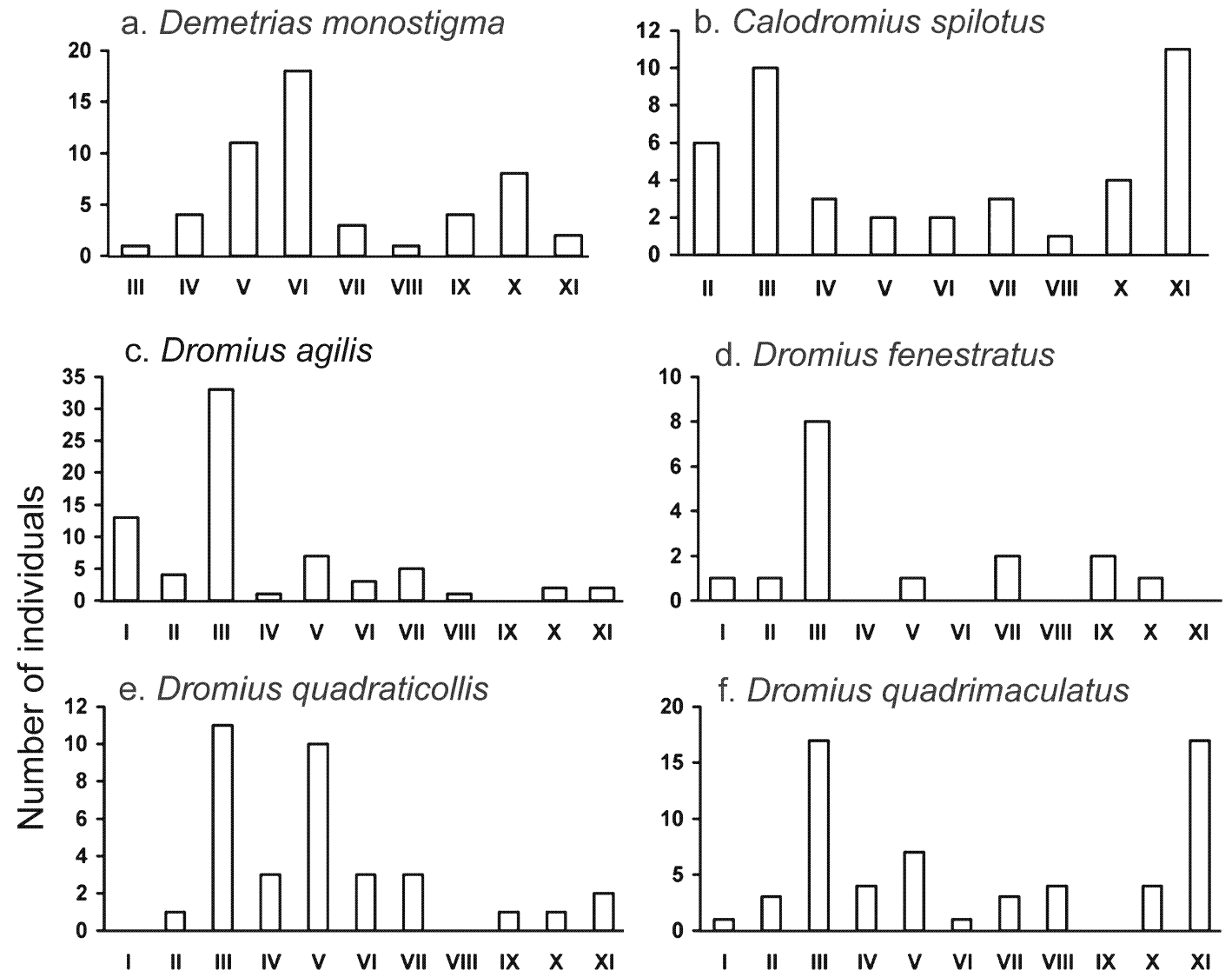

g. Dromius schneideri

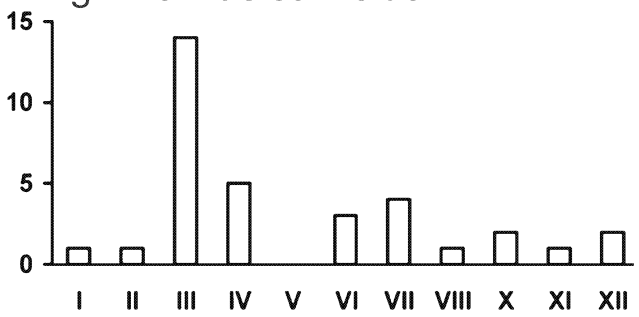

h. Microlestes minutulus

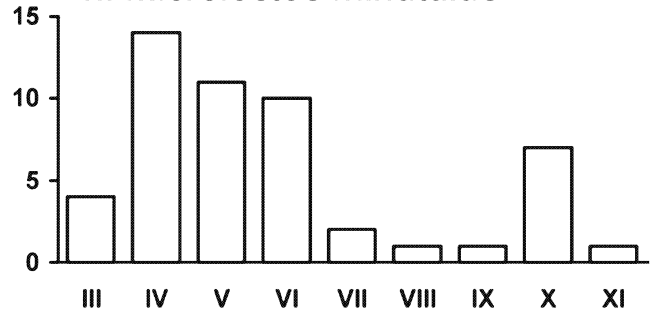

Month

Fig. 2. Monthly observations of adults of selected Lebiinae species in Lithuania.

16.V.2000, (1, leg. V. M.); Kaiš: Pravieniškès env., 26.V.2007 (1, leg. A. M.); Kau: Jiesia Land. R., 31.X.1983 (1, leg. R. F.), Kleboniškis f., 09.II.2001 (1, under bark of Pinus sylvestris, leg V. T.), Kamša B.-Z. R., 16.II.2001 (1, under bark of $P$. sylvestris, leg V. T.), Braziūkai f., 11.II.2011, 03.III.2012 (1, 1, under bark of $P$. sylvestris, leg V. T.); Vilemai env., 11.XI.2012 (4, under bark of $P$. sylvestris, leg V. T.), Kaunas t., "Panemunès šilas" park, 22.III.1992, 29.III.
1994, 04.XI.2001 (1, 2, 1, leg. R. F.); Kup: Vainiūniškis env., 01.IV.1993, 09.III.1997 (1, 1, leg. R. P.); Maž: Renavas env., 21.VII.1992 (1, leg. V. M.); Ner: Juodkrante env., 02.X.1991 (1, leg. B. Š.), Nida env., 20.VIII.1998 (1, leg. B. Š.); Pal: Šventoji env., 27.-28.VII.2008 (2, dunes, leg. A. B. \& K. B.); Plu: Grigaičiai env., 08.VI.1998 (1, leg. A. G.); Šak: Juškinè f., 11.XI.2001, 14.XI.2010 (4, 2, leg. R. F.); Šiau: Slydžiai env., 09.III.2001 (1, under bark of $P$. 
sylvestris, leg V. T.); Sila: Kaltinénai env., 02.III.2008 (1, leg. R. F.); Ukm: Dukstyna Ent. R., 30.X.1983 (1, leg. L. T.), 13.IV.1984 (1, leg. R. D.); Var: Marcinkonys env., 11.VI.1989 (1, leg. A. M.).

Earlier records. Only two earlier records are known: Vilnius town and Kaunas district (Pileckis \& Monsevičius 1982, Ferenca 1988). It was also noted for Klaipèda (Memel) environs without definite data (Bercio \& Folwaczny 1979).

General distribution. Europe, Caucasus, Algeria, Asia Minor. Not indicated for Lithuania in Catalogue of Palaearctic Coleoptera (Kabak 2003).

Notes. This dendrophilous species lives on trees, almost exclusively on pines. It is widely distributed in Lithuania, frequently found (Fig. 2b) and hibernating under bark (bark chips) of pines trunks.

Genus Dromius Bonelli, 1810

Subgenus Dromius Bonelli, 1810

D. (D.) agilis (Fabricius, 1787)

Examined material. Akm: Kamanos Nat. R., 22.X.1984, 31.VII.2004 (1, 1, leg. V. M.); Jurb: Liepgiriai f., 07.VI.1971 (1, leg. V. G.), 31.V. 1977, 02.VIII.1977, 29.V.1980, 27.VII.1983 (1, 1, 1, 1, leg. B. J.); Kaiš: Pravieniškès env., 23.V.1993 (1, leg. A. M.), Strévininkai f., 18.V.2003 (1, under bark of Quercus robur, leg. R. F.); Kau: Jiesia Land. R., 31.X.1983 (1, leg. R. F.), Dubrava f., 09.I.1983, 01.V.1989, 11.XI. 2011 (12, 1, 1, under bark of Pinus sylvestris, leg. R. F.), Dubrava f., 26.IV.2010 (1, pheromone trap for Ips typographus, leg. A. G.), Kamša B.-Z. R., 24.III.2000, 16.II.2001 (3, 2, under bark of $Q$. robur, leg. V. T.), Kleboniškis f., 16.I.2003 (1, under bark of $P$. sylvestris, leg V. T.), Braziūkai f., 20.III.2011, 25.III.2011 (1, 1, under bark of Larix decidua, leg V. T.), 03.III 2012 (1, under bark of $P$. sylvestris, leg V. T.), Kaunas t., "Vytautas" park, 12.VII.2010 (1, climbing on the trunk of $Q$. robur, leg. A. M.); Kup: Mirabelis f., 07.III.1992 (1, leg. R. P.), Siauriai env., 26.II. 1997 (1, leg. R. P.); Maž: Šernyné Telmological R., 14.XI.2000 (1, leg V. M.); Ner: Pervalka env., 03.VI.1995 (1, leg. B. Š.); Šak: Juškinė f., 09.II.2002 (1, leg. R. F.); Šiau: Rekyva f.,
20.III.2007 (9, leg. E. R.), Šiauliai t., Salduvė park, 09.III.2007 (16, leg. E. R.), Slydžiai env., 09.III.2001 (1, under bark of $P$. sylvestris, leg V. T.), Dzidai env., 04.V.2013 (1, trunk hollow of Fraxinus excelsior, leg. V. T.).

Earlier records. Only a few earlier records of this species are known: Vilnius and Panevėžys districts (Ogijewicz 1933, Ostrauskas \& Ferenca 2010). It was also noted for Kamanos Nature Reserve without definite data (Monsevičius 1997).

General distribution. Europa, West Siberia.

Notes. This dendrophilous species lives on deciduous as well as on coniferous trees. It is widely distributed in Lithuania, frequently found, especially in March (Fig. 2c), hibernates under bark (bark chips of trunks) of pines and oaks.

D. (D.) fenestratus (Fabricius, 1794)

Examined material. Kaiš: Vekonys env., 15.VII.1979, (1, leg. A. M.); Kau: Dubrava f., 09.I.1983 (1, leg. R. F.), Pajiesys f., 22.III.1995 (1, leg. R. F.); Kup: Vizgiūnai env., 31.III.1991 (1, leg. R. P.), Palėvenèlè env., 26.II.1997 (1, leg. R. P.); Mol: Kertuojai env., 05.V.2000 (1, pheromone trap for Ips typographus, leg. P. Z.); Rok: Pandèlys env., 30.VII.2008 (1, leg. A. B.); Šiau: Bacaičiai f., 21.III.1981 (1, leg. V. M.), Rèkyva f., 20.III.2007 (2, leg. E. R.); Ut: Antalgé env., 25.III.1977 (1, leg. A. K.).

Earlier records. Only a single earlier record is known: Švenčionys district (Šablevičius 2003). It has been also noted for Kamanos Nature Reserve and Klaipéda (Memel) environs without definite data (Bercio \& Folwaczny 1979, Monsevičius 1997).

General distribution. Europe. Not indicated for Lithuania in Catalogue of Palaearctic Coleoptera (Kabak 2003).

Notes. This dendrophilous species lives mostly on coniferous trees, rarely on deciduous ones. It seems to be the rarest species of the genus in Lithuania, everywhere sporadic. Half of the records are from March (Fig. 2d). Hibernating adults could be found mostly under bark (bark chips) of pines trunks.

D. (D.) quadraticollis Morawitz, 1862

Examined material. Akm: Kamanos Nat. R., 01.VI.1991, 16.IV.2000 (1, 1, leg. V. M.); Jona: Makštava f., 25.II.2014 (1, leaf litter of conifer- 
ous f., leg. V. T.); Jurb: Liepgiriai f., 23.V.1979 (1, leg. B. J.); Kaiš: Strévininkai f., 18.V.2006 (1, leg. R. F.); Kau: Dubrava f., 07.V.1996, 26.IV.2010, 02.VI.2010 (1, 2, 1, all caught by pheromone traps for Ips typographus, leg. A. G.), Gervénupis env., 30.V.1998 (1, leg. A. M.), Jiesia Land. R., 13.IX.1994 (1, leg. R. F.), Braziūkai f., 21.VII.2005 (1, leg. V. T.), 25.III.2011 (5, under bark of Larix decidua, leg V. T.); Kaunas t., "Panemunès šilas" park, 04.XI.2001 (1, leg. R. F.); Klai: Šernai env., 13.V.1989 (1, leg. S. K.); Kup: Drulènai env., 02.XI.1990 (1, leg. R. P.), Siauriai env., 26.II.1997 (1, leg. R. P.); Mol: Rudesa f., 20.V.1999 (1, pheromone trap for Ips typographus, leg. P. Z.); Ner: Juodkrante env., 31.III.2011 (1, leg. V. T.); Rok: Vosynos env., 05.V.2004 (1, leg. R. F.); Šak: Juškinė f., 06.X.1984 (1, leg. R. F.); Šiau: Slydžiai env., 08.III.2008 (1, in the hollow of Quercus robur, leg V. T.), Rèkyva f., 20.III.2007 (3, leg. E. R.), Šiauliai t., Salduvé park, 09.III.2007 (1, leg. E. R.); Ut: Minčia f., 25.V.2013, 10.VI.2013 (1, 1, window traps, leg. V. T.); Var: Puvočiai env., 17.V.2012 (1, leg. V. T.); Viln: Vilnius t., Verkiai f., 02.IV.1978 (1, leg. G. Š.), Vilnius t., Paneriai f., 14.IV.1979 (1, leg. S. P.), 30.V.1979 (1, leg. P. I.).

Earlier records. Only a single earlier exact record is known: Panevėžys district (Ostrauskas \& Ferenca 2010). It has been also noted for Kamanos and Viešvile Nature Reserves without definite data (Monsevičius 1997).

General distribution. Europe, Siberia. Not indicated for Lithuania in Catalogue of Palaearctic Coleoptera (Kabak 2003).

Notes. This dendrophilous species lives mostly on coniferous trees, rarely on deciduous ones. The species is widely distributed in Lithuania, with more than half of the records from March and May (Fig. 2e). It is frequently found hibernating under bark (bark chips) of various coniferous trunks.

D. (D.) quadrimaculatus (Linnaeus, 1758)

Examined material. Bir: Biržai t., 30.VII.2008(1, leg. A. B.); Ign: Ginučiai env., 16.VIII.1994 (1, under bark of Quercus robur, leg. B. Š.); Kaiš: Kruonis env. 13.VII.1996 (1, leg. A. M.), Strévininkai f., 26.III.2001 (1, under bark of $Q$. robur, leg. V. T.); Kau: Jiesia Land. R., 31.X.
1983, 23.III.1985, 24.I.1993; 27.III.1994 (2, 1, 1, 2, leg. R. F.), Pajiesys f., 07.V.1992 (1, leg. R. F.); Akademija env., 25.IV.1996 (1, under bark of Malus domestica, leg. V. T.), Kamša B.-Z. R., 24.IV.2000, 16.II.2001 (1, 2, under bark of $Q$. robur, leg. V. T.), Ežerélis env., 11.XI.2004 (1, under bark of Tilia cordata, leg. V. T.), Braziūkai f., 15.VI.2004, 19.VIII.2005 (1, 1, climbing on the branches of Q. robur, leg. V. T.); Kaunas t., Aleksotas, 18.XI.2001 (1, under bark of Q. robur, leg. V. T.), Kaunas t., Veršvai B.-Z. R., 14.VIII.2004 (1, leg. R. F.); Kup: Drulènai env., 20.X.1995 (1, leg. R. P.), Pamarnakiai env., 26.II.1997 (1, leg. R. P.); Kupiškis t., 11.XI.1990 (1, leg. R. P.); Maž: Gyvoliai env., 26.XI.1998 (1, leg. P. P.); Ner: Smiltyné env., 17.IV.1989, 25.VIII.1990 (1, 1, leg. S. K.), Curonian Spit env., 04.VIII.2000 (1, leg. B. S̆.); Rad: Radviliškis railway station env., 15.VII.2002 (1, multiple funnel trap baited with Alpha-Pinene, leg. H. O.); Šiau: Paežeriai env., 03.XI.1979 (1, leg. V. M.), Dzidai env., 25.V.1994 (1, climbing on the trunk of T. cordata, leg. V. T.), Bubiai env., 30.III.2000 (1, leg. V. T.); Rèkyva f., 20.III.2007 (9, leg. E. R.), Šiauliai t., Salduvé park, 09.III. 2007 (2, leg. E. R.), Slydžiai env., 08.III.2008 (1, in hollow of $Q$. robur, leg V. T.); $U t$ : Antalgé env., 17.IV.1977 (1, leg. A. K.); Var: Musteika env., 20.IV.1995 (1, leg. H. O.).

Earlier records. Only a few earlier records are known: Vilnius and Ignalina districts (Ogijewicz 1933, Ferenca 2004, Žiogas \& Zolubas 2005). It has also been noted for Kamša B.-Z. R. without definite data (Tamutis \& Dapkus 2008).

General distribution. Europe, Caucasus.

Notes. This dendrophilous species lives almost exclusively on deciduous trees. It is the most frequent species of the genus in Lithuania and it is widely distributed in forests and parks. It is frequently found under bark (bark chips), hollows and chaps of various deciduous trunks during non-vegetation period (Fig. 2f).

D. (D.) schneideri Crotch, 1871

Examined material. Akm: Kamanos Nat. R., 16.IV.2000 (1, leg. V. M.); Drus: Druskininkai env., 22.VI.1995 (1, on brances of Pinus sylvestris, leg. V. T.); Ign: Jonalaukis f., 10.IV.2000 (Šablevičius 2003, as Paradromius linearis), Kiauneliškis env., 08.IV.1997 (1, leg. B. Š.); 
Jona: Jonava t., 30.III.1976 (1, leg. S. Pa.), Makštava f., 25.II.2014 (1, leaf litter of coniferous f., leg. V. T.); Jurb: Liepgiriai f., 31.III.2011 (1, under bark of P. sylvestris, leg V. T.); Kau: Dubrava f., 08.III.1982, 09.I.1983 (5, 1, leg. R. F.), Kačerginè f., 01.IV.2001 (1, under bark of $P$. sylvestris, leg V. T.), Kleboniškis f., 09.II.2001 (1, under bark of $P$. sylvestris, leg V. T.), Braziūkai f., 03.III.2012 (1, bark of P. sylvestris, leg V. T.); Vilemai env., 11.XI.2012 (1, under bark of $P$. sylvestris, leg V. T.), Kaunas t., "Panemunès šilas" park, 29.III.1994, 25.XII.2004 (3, 2, under bark of P. sylvestris, leg R. F.); Kup: Mirabelis f., 07.III.1992 (1, leg. R. P.); Šiau: Slydžiai env., 09.III.2001 (1, under bark of $P$. sylvestris, leg V. T.); Šven: Kukliai f., 02.IV.2001 (Šablevičius 2003, as $P$. linearis); Ut: Minčia f., 06.I.2014 (1, leaf litter of coniferous f., leg. V. T.).

Earlier records. Reported from several Lithuanian districts: Kaunas, Ukmerge, Ignalina, Plungè, Varéna, Jurbarkas, and Vilnius (Ogijewicz 1933, Dvilevičius et al. 1988, Žiogas \& Gedminas 1994, Ferenca 2006).

General distribution. Europe, West Siberia, Kazakhstan.

Notes. This dendrophilous species lives mostly on coniferous trees, especially pines, less often on spruce or larch. It is a frequent species of the genus widely distributed in Lithuanian coniferous forests. Hibernating adults frequently occur under bark (bark chips) of pine trunks, especially in the southern exposition from September to April (Fig. 2g).

Genus Microlestes Schnmidt-Göbel, 1846

M. maurus maurus (Sturm, 1827)

Examined material. Kau: Noreikiškès env., 10.IV.2004 (1, dry meadow, leg. V. T.).

Earlier records. Only two old previous records in Lithuania: Vilnius and Panevéžys districts (Mazurowa \& Mazur 1939, Ferenca 2006).

General distribution. Europe, Asia Minor, Near East, Caucasus, Kazakhstan, Kirgystan. Not indicated for Lithuania in Catalogue of Palaearctic Coleoptera (Kabak 2003).

Notes. This species lives in open, dry habitats, and seems mostly to prefer sunexposed situations with sparse vegetation of herbs. It seems to be very rare in Lithuania. One previous record of this species from Curonian Spit (Žiogas \& Zolubas 2005) was based on missindentification and it is herein related to $M$. minutulus.

\section{M. minutulus (Goeze, 1777)}

Examined material. Kau: Akademija env., 10.IV.1994 (2, leg. V. T.), Margininkai env., 28.IV.1999, 03.V.1999, 23.V.1999, 27,VII.1999 (2, 1, 1, 1, tillage, leg. V. T.), Braziūkai env., 16.V.2002，23.IV.2007，05.III.2008，05.IV. 2010, 17.IV.2010, 11.IV.2011 (1, 5, 1, 1, 2, 3, dry meadows, leg. V. T.); Braziūkai f., 15.X.2010 (1, litter of coniferous forest (Piceetum oxalidosum), leg. V. T.), Kaunas t., "Ažžuolynas" park, 05.IX.2007 (1, leg. A. M.); Klai: Smiltynè env., 04.VII.1997 (1, leg. A. M.), 22.VI.2007 (1, leg. R. F.) 28.IV.2008, 04-11.V.2008 (5, 2, leg. P. I.); Mar: Menkupiai env., 05.X.1999 (4, leg. V. M.), Liubavas env., 05.X.1999 (1, leg. V. M.), Trakènai env., 03.XI.1999 (1, leg. V. M.); Ner: Juodkranté env., 27.V.1998 (1, leg. A. G., noted as Microlestes maurus by Žiogas and Zolubas (2005)); Nagliai Nat. R., 18.X.2005 (1, leg. P. I.); Grobštas Nat. R., 22.V.2006 (1, leg. R. F.); Pal: Palanga t., 05.VII.1996 (1, leg. V. T.); Plu: Peklinė f., 28.V.1971, 09.VI.1971 (1, leg. B. J.); Šak: Juškinè f., 12.III.2007 (1, leg. R. F.); Šiau: Slydžiai env., 03.V.1992 (1, pine forest, leg. V. T.), 16.VIII.1997 (1, tillage, leg. V. T.), Rejkyva f., 20.III.2007 (2, leg. E. R.); Šven: Baluoša env., 25.V.2001 (1, leg. R. F.); Zar: Yliškès env., 23.V.2001 (1, leg R. F.)

Earlier records. There are just a few previous records in Lithuania: Alytus, Lazdijai and Kaunas districts (Sharova \& Grüntal 1973, Tamutis 2000).

General distribution. Europe, Asia Minor, Caucasus, Central Asia, Siberia, Far East, Japan. Not indicated for Lithuania in Catalogue of Palaearctic Coleoptera (Kabak 2003).

Notes. This xerophilous species lives in various open areas: tillage, grasslands, forest edges and dunes. It occurs also in dry forest clearings and parks, and it is sometimes found under bark at the base of trees. It is a spring breeder and hibernates as an adult. According to our observations, Microlestes minutulus is quite common and widely distributed in Lithuania, generally found in open, dry, sandy or loamy habitats. Numeruos 
specimens were collected by pitfall traps on a sandy area in Curonian Spit as well as in tillage at Kaunas environs from April to June (Fig. 2h).

Genus Paradromius Fowler, 1887

Subgenus Manodromius Reitter, 1905

P. (M.) linearis linearis (Olivier, 1795)

Examined material. Drus: Gerdašiai env. 09.VIII.2002 (1, leg. R. F.); Kaiš: Kruonis env. 03.VI.2005 (1, leg. A. M.); Kau: Margininkai env., 07.XI.1999 (1, leg. V. T.), Ežerélis env., 24.III.2005 (1, exploited peat bog, leg. V. T.), Braziūkai env., 12.VI.2004, (1, dry meadow, leg. V. T.), 13.IX.2005 (1, dry meadow, leg. V. T.), 20.VII.2007 (2, dry meadow, leg. V. T.), 05.IV.2010 (1, dry meadow, leg. V. T.), Kaunas t., Aleksotas env. 29.VII.2004 (1, leg. A. M.); Mar: Juodeliai env. 30.V.2002 (1, leg. R. F.); Pal: Monciškė env., 09.V.2001 (1, leg. R. F.); Šiau: Slydžiai env., 04.V.2013 (2, dry meadow, leg. V. T.); Var: Merkinè env., 13.VII.1999 (1, leg R. F.).

Earlier records. Only a few earlier records are known: Baltic sea coast (Barševskis 2001, Ferenca et al. 2002), Ignalina, Švenčionys, and Vilnius districts (Šablevičius 2003, Ferenca et al. 2006).

General distribution. Europe, Northern Africa, Caucasus, Asia Minor, Near East, Turkmenistan, Kirgyzstan. Not indicated for Lithuania in Catalogue of Palaearctic Coleoptera (Kabak 2003).

Notes. According to Lindroth (1992), this is a markedly xerophilous and heat-requiring species. According to our observation it is quite common in open, dry, sandy habitats, especially in unmanaged grasslands on Calamagrostis epigejos overgrowths and on dune seashores. It is widely distributed in Lithuania. The previous records on this species, found under the bark of Pinus sylvestris (Šablevičius 2003), were based on wrong identification and should be related to Dromius schneideri. So, we cannot confirm the relation of this species with trees. However, in literature survey this species is mentioned as a trunk dweller (Felix \& Wielink 2011). The adults were found from March to November (Fig. 3a).
Subgenus Paradromius Fowler, 1887

P. (P.) longiceps (Dejean, 1826)

Examined material. Ner: Juodkrantè env.

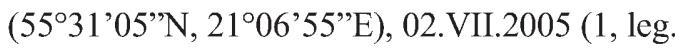

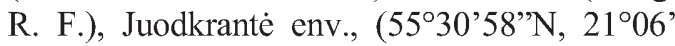
57'E), 06.VII.2012 (1, leg. V. T.).

Earlier records. The earlier record of this species from Lithuania (Tamutis et al. 2008) was erroneous and related to $D$. atricapillus (see above). This species is also noted for Lithuania in the Catalogue of Palaearctic Coleoptera (Kabak 2003) and Fauna Europaea Web Service (Vigna Taglianti 2012), but the primary sources of these notifications remain unknown for the authors.

General distribution. Europe, Caucasus.

Notes. Although the records of the species are not many in Lithuania, it seems to mainly occur on the coastal dunes, living in the fascicles of grasses (Elymus, Ammophila, Calamagrostis).

Genus Philorhizus Hope, 1836

Ph. sigma (Rossi, 1790)

Examined material. Akm: Kamanos Nat. R., 19.X.1984, 07.XI.1987, 17.VII.1993，28.IX. 1997 (1, 1, 1, 1, leg. V. M.), 04.V.1992 (1, leg. R. F.) Gimbetiškė env., 09.V.1995 (4, leg. V. M.), Klyšiai env., 12.IV.1993 (5, leg. V. M.), Alkiškiai quarry territory, 07.IV.2012 (1, under plant debris close to water stand, leg. V. T.); Bir: Biržai t., 30.VII.2008 (1, leg. A. B.); Kaiš: Pravieniškès env., 31.VII.1985 (1, leg. R. F.), Būda B.-Z. R., 08.VIII.1996 (1, leg. R. F.); Kau: Dubrava f., 19.IV.1982, 16.IV.1990, 01.V.1990, 05.IV.1992 (1, 1, 1, 2, leg. R. F.), Novaraistis Orn. R., 24.III.2007, 08.IV.2011 (2, 1, under plant debris, leg. V. T.), Ringové Ent. R., 20.V.1998 (1, leg. R. F.), 04.VIII.2009 (1, leg. V. T.); Pajiesys f., 28.III.2000 (2, leg. R. F.), Jiesia Land. R., 22.VI.1991 (1, leg. A. M.), Noreikiškès env., 23.VIII.1995 (noted as Philorhizus notatus by Tamutis et al. (2008)), Margininkai env., 21.IV.1996 (noted as Philorhizus notatus by Tamutis et al. (2008)), 29.VIII.1997, 07.IV.2000 $(1,1$, Carex sp. overgrowth, leg. V. T.), Braziūkai env., 03.IV.2011, 25.III.2012 (1, 1, under plant debris on forest edge, leg. V. T.), 16.IV.2012, 26.IX.2012 (2, 2, leaf litter of osier-bed, leg. V. T.); Kup: Vizgiūnai env., 06.IV.1991 (1, leg. R. 

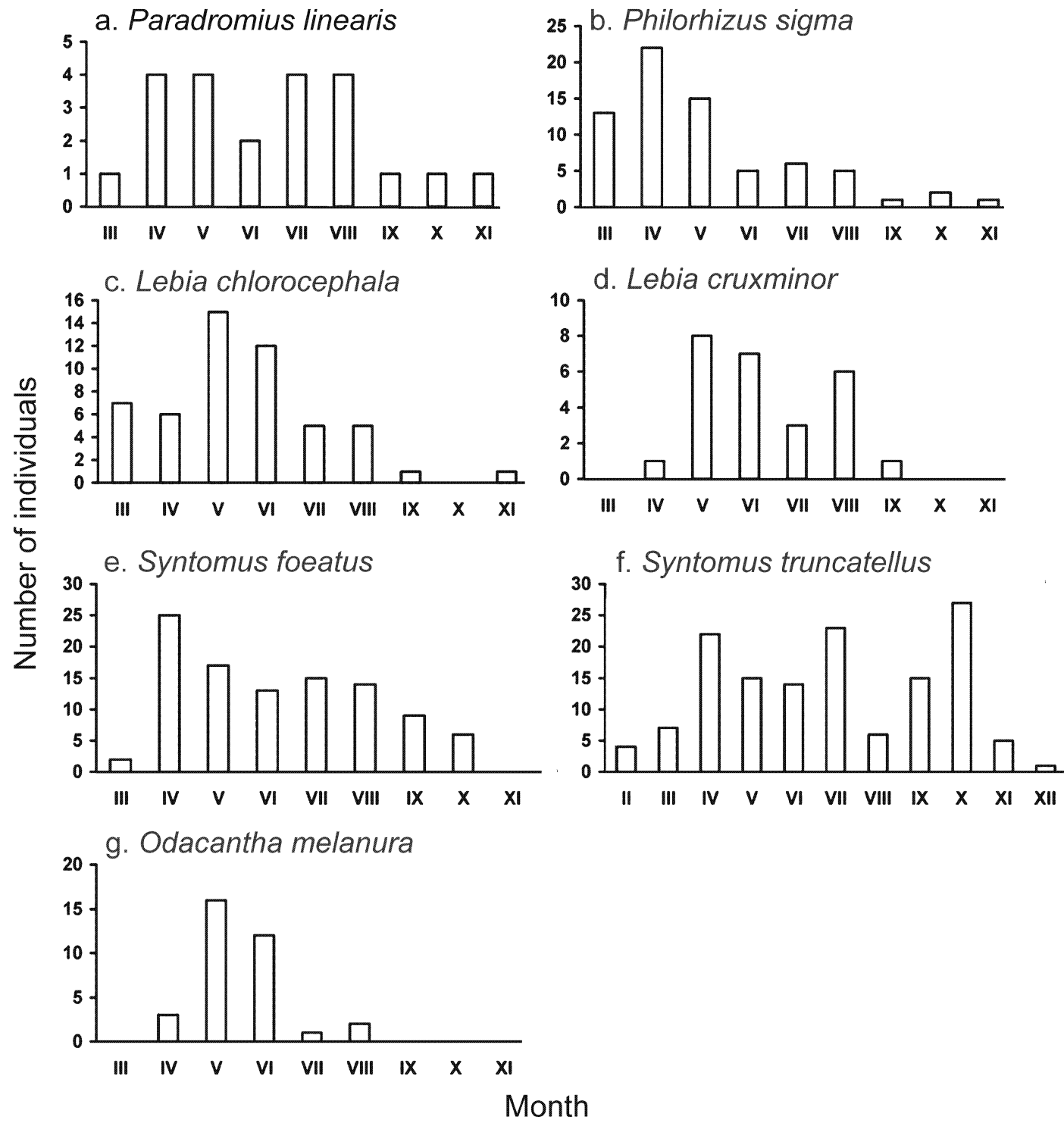

Fig. 3. Monthly observations of adults of selected Lebiinae species in Lithuania.

P.); Mar: Orija env., 05.V.2000 (1, leg. V. M.), Liudvinavas env., 05.V.2000 (1, leg. V. M.); Ner: Smiltynė env., 27.V.1990 (1, leg. S. K.), Alksnynè, env., 03.VI.2000 (1, leg. B. Š.), Juodkrantė env., 05.VI.2001, 10.VI.2002 (1, 2, leg. B. Š.), 02.VII.2005 (2, leg. R. F.), Lapnugaris Land. R., 27.V.2010 (2, leg. R. F.); Pal: aeroport env., 30.V.2001 (1, leg. R. F.); Šak: Paluobiai, 27.III.2010, 31.X.2011 (4, 1, leg. R. F.); Šiau: Dzidai env., 16.VIII.1997 (1, bank of pond, leg. V. T.), 27.IV.1996 (3, moist meadow, under plant debris, leg. V. T.), 10.III.2001 (2, leaf litter of osier-bed, leg. V. T.), Slydžiai env., 08.V.1992 (2, dry meadow, under plant debris, leg. V. T.), Rèkyva lake env., 20.III.2007 (1, leg. E. R.).

Earlier records. The earlier data on the distribution of this species in Lithuania was actually very poor. Without definite finding data, this species was noted from Kamanos, Žuvintas and Čepkeliai Nature Reserves (Monsevičius 1997). Two previous records of Philorhizus notatus (Stephens, 1827) were based on misidentification (Tamutis et al. 2008) and herein related to Philorhizus sigma. So, Ph. notatus has not been 
found in Lithuania yet and we suggest removing it from the list of Lithuanian beetles.

General distribution. Europe, Asia Minor, Near East, Caucasus, Siberia. Not indicated for Lithuania in Catalogue of Palaearctic Coleoptera (Kabak 2003).

Notes. This hydrophilic species generally lives in moist habitats or swamps, richly and thick overgrown by grasses, but it is also not rare in dry, sandy habitats. By assessing new examined material, $P h$. sigma was judged as widely distributed in Lithuania, frequently found in moist and dry habitats, richly overgrown by grasses: fresh water shores and meadows, especially at places where Salix shrubs border with grasslands. The adults were found from March to November (Fig. 3b).

\subsubsection{Subtribe Lebiina Bonelli, 1810}

Genus Lebia Latreille, 1802

Subgenus Lamprias Bonelli, 1810

L. (L.) chlorocephala (J. J. Hoffmann, 1803)

Examined material. Anyk: Katleriai env., 06. VIII.1988 (1, leg. S. K.), Biténai, 02.III.1997 (2, leg. R. P.); Kaiš: Kruonis, 03.V. 1998 (1, leg. A. M.), Morkūnai, 21.VIII.2002 (1, meadow, leg. V. T.); Kau: Romainiai, 07.IV.1966 (1, leg. E. G.); Jiesia Land. R., 27.V.1984 (1, leg. R. F.); Dubrava f., 16.VIII.1986 (1, leg. R. F.); Pypliai env., 31.III.2002 (1, natural meadow, leg. V. T.); Noreikiškès env., 03.III.1994 (1, wet meadow, V. T.), Akademija env., 03.V.2000 (1, meadow, V. T.), 06.VI.1999 (1, leg. V. T.); Kaunas t., Panemunès šilas f., 06.V.2004 (1, clearing, leg. V. T.); Karmélava env., 21.V.2007 (1, leg. V. I.); Braziūkai env., 27.IV.2011 (1, dry meadow, leg. V. T.) Braziūkai env. 14.VII.2012 (1, garden, leg. V. T.), Girionys env. 02.VIII.2012 (1, leg. K. L.); Klai: Pèžaičiai env., 17.VI.1995 (1, leg. S. K.); Kre: Monciškè env., 09.V.2001 (1, leg. R. F.); Kup: Drulènai env., 18.XI.1990 (1, lakeside, leg. R. P.); K.R.: Kazlų Rūda env., 26.III.1996 (1, leg. E. G.); Ras: Ariogala env., 13.IX.1979 (1, leg. A. Š.); Rok: Obeliai env., 30.VII.2008 (1, leg. A. B.); Šiau: Meškuičiai env., 05.VI.2005 (1, leg. anonymous); Šila: Milaičiai env., 27.IV.2013 (1, leg. R. F.); Ukm: Dukstyna Ent. R., 07.IV.1985 (1, leg. R. D.); Ut: Utena t., 13.VI.1965 (1, leg. E. G.);
Antalgé env., 30.III.1977, 10.IV.1978 (1, 1, leg. A. K).

Earlier records. In Lithuania, the species was earlier recorded in Vilnius (Ogijewicz 1933, Ivinskis et al. 2009), Palanga (Ferenca et al. 2002), Klaipèda (Ferenca et al. 2006), Anykščiai, Ignalina, Šilalè, Švenčionys, Neringa (Šablevičius 2003), and Kaunas districts (Inokaitis 2004).

General distribution. Europe, West Siberia, Kazakhstan, eastern Sayanin.

Notes. This mezophilic species prefers unshaded habitats: grasslands, meadows and pastures. The larvae attacked mature larvae and freshly formed pupae of the leaf beetle Chrysolina varians (Lindroth, 1954). The largest numbers of individuals was collected in May and June (Fig. 3c), usually observed running on soil surface in various types of meadows or have been caught using sweep net. In March, April, September, and November the specimens of this species were occasionally found under plant debris. Our observations confirm the notion of other authors that the peak activity of the adults of this species is in May and June and that it overwinters in the adult stage (Aleksandrowicz \& Maksimenkov 1994).

\section{L. (L.) cyanocephala (Linnaeus, 1758)}

Examined material. Trak: Varnikai env., 07.X.1929 (1, under stone, leg. B. O.); Viln: Vilnius t., Šeškinè env., 19.VIII.1923 (2, under stone, leg. B. O.).

Earlier records. Above mentioned records are the single ones in Lithuania, published 80 years ago (Ogijewicz 1933).

General distribution. Europe, Northern Africa, Near East, Asia Minor, Caucasus, Central Asia, southern West Siberia, northwestern China.

Notes. This mostly xerophilic, herbobiontic species prefers open habitats: grasslands, meadows and forest edges. The larvae attacked mature larvae and freshly formed pupae of the leaf beetles Chrysolina hyperici and Ch. marginata (Sazhnev \& Eskov 2010). The species is sporadic and locally distributed throughout all its distribution range, especially sparse in the northern part (Haberman 1968, Aleksandrowicz 1991, Barševskis 2003, Alekseev 2008, Karjalainen et al. 2010, Ljunberg et al. 2010). There are no new findings in the Western lowland forest zone of 
Russia for more than 80 years (Aleksandrowicz 1993). Considering these facts, it could be argued that this species is endangered or probably extinct in these regions and in Lithuania as well. In our opinion, the main limiting factor for survival of this species is the scarce presence of its hosts in the region (Lopatin \& Nesterova 2005, Bukejs \& Telnov 2010, Bukejs et al. 2011).

Subgenus Lebia Latreille, 1802

\section{L. (L.) cruxminor (Linnaeus, 1758)}

Examined material. Akm: Kamanos Nat. R., 31.V.2002 (1, leg. V. M.); Anyk: Burbiškis env., 10.VII.1994 (1, leg. S. K.); Kau: Jiesia Land. R., 25.IV.1982 (1, leg. R. F.); Braziūkai env., 12.VI.2003 (1, moist forest edge, on Salix sp., leg. V. T.), 18.VII.2008 (1, dry meadow, leg. V. T.), 12.V.2010, (1, dry meadow, leg. V. T.); Karmèlava env., 16.VI.2006 (1, leg. V. I.); Girionys env., 02.VIII.2012, (1, leg. K. L.); Kup: Užubaliai env., 05.V.1995 (1, leg. R. P.); Maž: Vilioté env., 27.V.1998 (1, leg. V. M.); Ner: Nida env., on the sand in the beach, 24.V.1991 (1, leg. V. T.); Pal: aeroport env., 28.VIII.2001 (1, leg. R. F.), Šventoji env., 27.VII.2008 (1, dunes, leg. A. B. \& K. B.); Šiau: Mirsiškè env., 02.VI.1996 (1, dry meadow, leg. V. T.); Šven: Obelų Ragas env., 05.VII.1979 (1, leg. S. Pa.), 21.V.1982 (1, leg. S. Pa.); Ukm: Dukstyna Ent. R., 08.V.1979 (1, leg. G. Š.); Ut: Antalgè env., 14.VI.1982 (1, leg. A. K.); Var: Marcinkonys env., 15.V.1984 (1, leg. R. F.), Viln: Vilnius t., Naujieji Verkiai, 05.VIII.1980 (1, leg. P. I.); Vilnius t., Paneriai, 01.-30.VIII.1980 (1, leg. K. A.).

Earlier records. In Lithuania, the speceis was earlier recorded in Vilnius (Ogijewicz 1933) and Kaunas (Inokaitis 2004) districts.

General distribution. Europe, Tunisia, Near East, Asia Minor, Caucasus, Central Asia, Siberia, Kazakhstan, Kirgyzstan, Mongolia, Far East, Japan.

Notes. This mezophilic, herbobiontic species, prefers unshaded habitats: grasslands, meadows and pastures. The larvae attacked mature larvae and freshly formed pupae of the leaf beetles Galeruca tanaceti, Chrysolina varians and $C$. hyperici (Wachman et al. 1995, Anderson 2006). The species is widely distributed in Lithuania but it is observed sporadically everywhere. The larg- est numbers of individuals were collected in May, June, and August (Fig. 3d), usually observed in various types of meadows.

\subsubsection{Subtribe Lionychina Jeannel, 1948}

Genus Lionychus Wissmann, 1846

Subgenus Lionychus Wissmann, 1846

L. quadrillum (Duftschmid, 1812)

Examined material. B.: Škevonys Geomorphological R., 17.VIII.2005 (11, in a sun-exposed gravel slope, leg. R. F.); Pal: Palanga aeroport env., 09.V.2001, 26.VII.2001 (5, 5, slope of open, gravelly, sand pit habitat, with sparce vegetation, leg. R. F.).

Earlier records. Above mentioned findings are so far the only published records of this species in Lithuania (Ferenca et al. 2002, 2006).

General distribution. Europe, Caucasus, Asia Minor, Israel. Not indicated for Lithuania in Catalogue of Palaearctic Coleoptera (Kabak 2003).

Notes. This xerophilic species occurs on unshaded, sandy or gravely habitats, especially water borders or slopes of sand pits, and prefers highly sun-exposed conditions. According to results of Swedish scientists, it was the most numerous carabid species in sand pits, highly sun-exposed habitats in the Uppsala County (Lönnberg \& Jonsell 2012). However, this species is still rare, reported only from 2 places in Lithuania.

\section{Genus Syntomus Hope, 1838}

S. foveatus (Geoffroy, 1785)

Examined material. Kaiš: Kruonis env., 06.IV.1975, (1, leg. A. M.), Pravieniškès env., 26.V.2007 (1, leg. A. M.); Kau: Dubrava f., 11.IV.1981, 08.III.1982, 02.VIII. 1983, (24.III., 07.IV., 02.VI., 07.VII.1985), 05.IV.1986 (1, 1, 1, 1, 7, 7, 2, 1, leg. R. F.); Ežerèlis env., 06.VIII. 1982, 26.IV.1986 (1, 1, leg. R. F.); Gaižènèliai 17.VIII.1998, 28.V.2000 (3, 1, leg. V. T.); Braziūkai f., 20.IV.2004, 01.IV.2007, 28.X. 2007, 28.IX.2012 (4, 1, 3, 2, forest clearings overgrown by Calluna and Thymus plants, leg. V. T.); Girininkai II env., 13.IX.1997 (1, heaths, leg. V. T.); Jiesios Land. R., 15.IV.1985 (1, leg. R. F.); K.R.: Jūrè env., 24.VIII.1984 (1, leg. R. F.), Kajackai env., 29.VII.1985 (1, leg. R. F.); Klai: Nemirseta env., May-July, 2004 (6, pitfall traps, 
leg. P. I.), 15.VI.2004, 15.V.2005 (3, 3 leg. R. F.); Mar: Juodeliai env., 15.VIII.2001 (1, leg. R. F.); Ner: Nagliai Nat. R., 26.V.2010 (1, leg. R. F.); Pal: Šventoji env., 27.VII.2008 (1, dunes, leg. A. B. \& K. B.); Prie: Palios peat bog, 28.IV.1999 (1, leg. D. D.); Šak: Lekéčiai env., 01.V.1988 (1, leg. R. F.); Šiau: Slydžiai env., 09.VII.1992, 16.VIII.1997, 24.V.2002, 04.VIII.2002, 10.VIII. 2002，01.IV.2004，05.IV.2010，14.IV.2012， 18.VII.2012 (1, 1, 2, 1, 1, 1, 1, 1, 2, dry meadow with sparce vegetation, leg. V. T.), Tau: Viešvilè Nat. R., 13.IX.2006 (2, leg. R. F.), Sakalinè f., 09.V.2008 (5, leg. V. T.); Ut: Antalgè env., 17.IV.1977, 19.X.1977, 19.IV.1978 (1, 1, 1, leg. A. K.); Var: Puvočiai env. 17.V.2012 (1, leg. A. A.).

Earlier records. Only old earlier records of this species are known: Kaunas, Panevėžys, and Vilnius districts (Ogijewicz 1933, Ferenca 2006). It has been also noted for Čepkeliai Nature Reserve without definite data (Monsevičius 1997).

General distribution. Europe, Caucasus, northern Africa, Cyprus, Syria, Kazakhstan, West Siberia.

Notes. This markedly xerophilous species occurs mainly in habitats of open, dry, sun-exposed, sand or gravel with sparce vegetation. It is also frequently found in peat bogs or forest clearings overgrown with Calluna or Thymus plants. After assessing new examined material it was concluded that $S$. foveatus is widely distributed in Lithuania, frequently found in open, dry, sand or gravel habitats. It is especially common in dry meadows with sparce vegetation and in forest clerings. The adults were found from March to October (Fig. 3e).

\section{S. truncatellus (Linnaeus, 1761)}

Examined material. Akm: Kamanos Nat. R., 31.V.2002 (2, leg.V. M.), Naujoji Akmenè t., 04.IV.2010 (1, in park, leg. V. T.), Alkiškiai gravel quarry, 07.IV.2012 (1, leg. V. T.); Bir: Tabokinè env., 14.VIII.1984 (1, leg. R. F.), Biržai t., 30.VII.2008 (2, leg. A. B.); Joni: Girkančiai env., 02.X.1990 (13, leg. V. M.); Klai: Smiltyné env., 11.IV.2004 (1, leg. R. F.); Kau: Braziūkai env., 20.IV.2004, 15.VI. 2004, 15.VII.2004, 29.IX.2004, 10.XII.2004, 08.V.2005, 10.V. 2006, 10.IX.2006, 21.III.2007, 10.VIII.2007, 25.XI.2007, 18.II.2008, 05.III.2008, 06.-12.IV.
2008, 26.IX.2009, 5.-17.IV.2010，11.IV.2011, 24.III.2012，16.V.2012，30.VIII.2012，23.IX 2012, 15.IV.2013 (3, 1, 1, 2, 1, 1, 1, 6, 2, 4, 2, 4, 1, $4,3,3,3,4,1,1,2,1$, in open places: tillage, meadows, forest clearings, leg. V. T.), Dubrava f., 15.V.1969 (1, leg. S. Pileckis), 30.VI.1984, 05.IV.1986 (1, 1, leg. R. F.), Ežerèlis env., 26.IV.1986 (1, leg. R. F.), 28.VI.2010 (1, leg. A. M.), Narépai env., 19.VII.2005 (1, leg. V. T.), Noreikiškès env., 16.V.2002 (1, leg. V. T.), Novaraistis Orn. R., 30.IX.2007 (3, leg. V. T.), 08.IV.2010 (1, leg. B. T.), Kaunas t., Pažaislis env., 27.V.2002 (2, leg. V. T.); Mar: Menkupiai env., 05.IX.1999, 05.X.1999 (1, 11, leg. V. M.), Trakènai env., 03.XI.1999 (2, leg. V. M.), Garbrieliškiai env., 03.XI.1999 (1, leg. V. M.), Juodeliai env., 27.VI.1995 (4, leg. H. O.); Ner: Juodkrante env., 10.VII.2000 (1, leg. R. F.); Pal: Šventoji t., 06.X.2001 (1, coastal dunes, leg. V. T.), Palanga t., 05.VII.1996 (3, coastal dunes, leg. V. T.); Prie: Palios peat bog, 30.VI.1999 (1, leg. D. D.); Rok: Obeliai env., 30.VII.2008 (2, leg. A. B.); Siau: Slydžiai env., 09.III.2001, 08.IV.2004, 19.VII.2012 (1, 1, 2, dry meadow, leg. V. T.), Einoraičiai env., 15.VII.1998 (3, leg. R. F.); Ukm: Pašilè f., 30.IV.2004 (1, leg. N. N.); Var: Merkiné env., 02.VII.1997 (1, leg. A. M.), Čepkeliai Nat. R., 19.V.2002 (1, leg. V. T.), Puvočiai env., 16.V.2012 (1, dry meadow, leg. V. T.); Zar: Salakas env., 27.VI.1996 (1, leg. H. O.).

Earlier records. This species has been reported from several Lithuanian districts: Vilnius, Ukmergé, Šiauliai, Alytus (Ogijewicz 1933, Mazurowa \& Mazur 1939, Monsevičius 1997, Dvilevičius et al. 1988, Ferenca et al. 2002).

General distribution. Europe, Caucasus, northern Africa, Cyprus, Syria, Kazakhstan, West Siberia.

Notes. This eurytopic species prefers open, dry, sandy, gravelly or peaty habitats, with more or less continuous vegetation or small subshrubs like Calluna, Thymus, etc. It could often be found hibernating in forest litter. In contrary to a previous view suggested by Pileckis and Monsevičius (1995), we consider the species as common and widely distributed in Lithuania. It is especially frequent in sandy, dry meadows where it has been numerously observed (Braziūkai and Slydžiai environs) almost all-year-round (Fig. 3f). 


\subsection{Tribe Odacanthini Laporte, 1834}

Genus Odacantha Paykull, 1798

Subgenus Odacantha Paykull, 1798

O. melanura (Linnaeus, 1767)

Examined material. Alyt: Bambininkai env., 27.V.1981 (1, leg. S. Pa.); Anyk: Rubikiai lake env., 06.VI.1992 (1, leg. S. K.); Bir: Nemunèlio Radviliškis env., 04.VIII.1997 (1, reed bed of riverside, leg. A. G.); Jurb: Viešvilè Nat. R., Smaladaržis env., 13.V.2008 (1, reed bed of riverside, leg. V. T.); Ner: Smiltynė env., 03,V.2000 (1. leg. S. K.); Ner: Juodkrantė env., 02.VII.2005 (1, reed bed of Curonian lagoon, leg. R. F.), 26.V.2011 (2, flying near coast of Curonian lagoon, leg. V. T.), Nagliai Nat. R., 27.V.2009 (1, reed bed of Curonian lagoon, leg. R. F.); Kaiš: Darsūniškis env., 06.V.1970 (1, reed bed of lakeside, leg. A. M.); Pravieniškès env., 06.V.1986 (1, leg. R. F.); Kau: Jiesia Lan. R., 26.V.1985 (1, reed bed of riverside, leg. R. F.); Noreikiškès env., 20.V.1994 (1, reed bed of the shore of pond, leg. V. T.); Gaižènèliai env., 18.V.2000 (1, reed bed of the shore of pond, leg. V. T.); Novaraistis Orn. R., 05.VI.2005 (1, reed bed of swamp, leg. V. T); Kup: Antašava env., 12.V.1995 (1, leg. R. P.), Vainiūniškis env., 27.V.1995 (1, reed bed of the shore of pond, leg. R. P.); Mol: Aisetas lakeside, 28.V.1996 (1, reed bed, leg. V. T.); Prie: Šilavotas env. 03.VI.2012 (1, silty, rush overgrowth channel, leg. V. T.); Rad: Praviršulis lakeside, 07.VI.1969 (1, reed bed, leg. A. J.); Šiau: Dzidai env., 15.V.1998 (1, reed bed of the shore of pond, leg. V. T.), Slydžiai env., 04.V.2013 (1, reed bed of swamp, leg. V. T.); Šla: Šilale t., 19.VI.1990 (1, reed bed of the shore of pond, leg. B. Š.); Šilu: Ventès Ragas env., 24.-26.VIII.1988 (3, reed bed of Curonian lagoon, leg. R. F. and S. G.); Ukm: Dukstyna Ent. R., 15.IV.1984 (1, leg. R. D.).

Earlier records. Central and Baltic Sea coast regions of Lithuania (Pileckis 1963, Zajančkauskas \& Pileckis 1968, Sharova \& Grüntal 1973, Ivinskis et al. 1998).

General distribution. Europe, Caucasus, Asia Minor, Iran, Kazakhstan, southwestern Altai.

Notes. This species occurs on the shores of eutrophic, Phragmites-rich waters but rarely could be found on the shores ranked with Typha latifolia, Glyceria spectabilis or Juncus sp. plants. It is a widely distributed species in Lithuania, almost exclusively found in reed beds on the shores of eutrophic, both running and standing waters. We have observed active adults during the warm, sunny days flying or climbing on stems of reeds or accasionally running on the ground surface. The obvious preference of this species to large reed beds as of Curronian lagoon was settled; on small ones, such as ponds and swamps, it was observed only occasionally. The adults were found from April to August (Fig. 3g).

\section{Discussion}

This paper is the first comprehensive review on the species composition, distribution, and some ecological features of Lebiinae ground beetles in Lithuania. During our study, the species composition of Lithuanian Lebiinae ground beetles was specified.

\subsection{Faunistics of Lithuanian species of Lebiinae}

We discovered that Lebiinae subfamily is presented by 25 species belonging to 11 genera and two tribes in Lithuania. The tribe Odocanthini is monotypical, presented by a single species. Lebiini tribe is more diverse, presented by four subtribes, ten genera and 24 species. The most numerous genera are Dromius (5 species) and Cymindis (4 species).

The record of Cymindis humeralis remains unconfirmed, because the evidential specimen was not available for the authors. The occurrence of this species in Lithuania is possible, albeit with the absence of the trustworthy records of this species in Latvia (Spuris 1983) and Estonia (Haberman 1968), and only solitary records from Belarus (Aleksandrowicz 1993), Kaliningrad region (Alekseev 2008), northern Poland (Burakowski et al. 1974) and southern Sweden (Ljunberg et al. 2010) clearly demonstrates the rarity of this species in the region.

The occurrence of Philorhizus notatus (Stephens, 1827) in Lithuania is disproved as the noti- 
fication of this species was based on erroneously identified specimens belonging to Philorhizus sigma. The expectation of occurrence of this species in Lithuania is quite problematic, because the information on its distribution in the northern $\mathrm{Eu}-$ rope is still contradictory.

There are not actual data on its occurrence in Latvia as earlier records (Barševskis 1987, Cibulskis 1997) were based on missidentified material and should be related to Ph. sigma (A. Barševskis pers. comm.). Trustworthy records of this species are still lacking in Belarus, too. The record from Vitebsk district (north-western Belarus) (Radkevich 1970) was probably based on missidentification (O. R. Aleksandrowicz pers. comm.). Philorhizus notatus is rare in northeastern part of Poland as well, but it is commonly found in southern Sweden and Finland (Lindroth 1992, Karjalainen et al. 2010).

It is important to note that Demetrias atricapillus, characteristic for Southern and Central Europe, was found in Lithuania for the first time during the current study. Unfortunately, more comprehensive information on the distribution of this species in Lithuania remains poor. Further north from Lithuania the species was recently recorded in Latvia (Telnov et al. 2005).

According to Gorodkov's (1984) description of types of distributional ranges of insects, Lebiinae ground beetles of Lithuania could be attributed to eight horotypes (Table 1). The ranges of 18 Lithuanian Lebiinae ground beetles species are limited to the western Palaearctic and ranges of only seven species cover the entire Eurasian continent.

We would like to pay attention to changes in the distribution of Lebia cyanocephala not only in our country but also throughout the whole northern part of the species' range. At the beginning of 20th century this species occured in Norway, Finland, Sweden, Latvia, Belarus and Lithuania (Ogijewicz 1933, Spuris 1983, Lindroth 1992, Aleksandrowicz 1993), but no more have been found in the above mentioned countries for more than eighty years. It is also interesting to note the opposite tendency. The species Lionychus quadrillum was known only in Southern and Western Europe until the 1970s, but over period of the past 35 years this species has reached the northern European countries such as Den-
Table 1. The spectrum of horotypes of Lithuanian Lebiinae (horotypes according Gorodkov 1984).

\begin{tabular}{|c|c|}
\hline Horotypes & Species \\
\hline Transpalearctic & $\begin{array}{l}\text { Lebia cruxminor } \\
\text { Microlestes minutulus } \\
\text { Philorhizus sigma } \\
\text { Syntomus foveatus } \\
\text { Syntomus truncatellus }\end{array}$ \\
\hline Transeurasian & $\begin{array}{l}\text { Cymindis vaporariorum } \\
\text { Dromius quadraticollis }\end{array}$ \\
\hline Western Palearctic & $\begin{array}{l}\text { Calodromius spilotus } \\
\text { Demetrias atricapillus }\end{array}$ \\
\hline
\end{tabular}

Western Central Palearctic

Demetrias imperialis

Lebia cyanocephala

Paradromius linearis

Euro-Sibero-Central Asiatic

Cymindis angularis

Cymindis macularis

Dromius agilis

Dromius schneideri

Lebia chlorocephala

Odacantha melanura

European

Cymindis humeralis

Euro-Caucasian

Dromius fenestratus Dromius quadrimaculatus Lionychus quadrillum

Paradromius longiceps

Euro-Kazakhstanian

Demetrias monostigma Microlestes maurus

mark, Sweden, Finland, Lithuania and Belarus (Burakowski et al. 1974, Clayhill 1984, Ferenca et al. 2002, Tsinkevich \& Aleksandrowicz 2002, Silfverberg 2010).

It is evident from the comparison of the species composition of the subfamily Lebiinae in Lithuania and neighbouring countries that it is not fully explored in Lithuania. Based on this assumption, we believe that the fauna of Lebiinae ground beetles in Lithuania will increase at least by another two species in the nearest future. It is most likely that Dromius laeviceps and Dromius angusticollis live in Lithuania, but that should be confirmed by actual data.

Summarizing the available faunistic data, it can be concluded that ground beetles belonging to the subfamily Lebiinae are distributed unevenly in Lithuania. More than half of the spe- 
Table 2. Ecological characteristics of Lithuanian Lebiinae (following Koch 1989, Lindroth 1992, Barševskis 2003 and our observations) (S: stenotopic, E: eurytopic, X: xerophilic, M: mezophilic, Hi: higrophilic, D: dendrobiont, Hr: herbicolous, Ep: epigeous, Ps: psammophilic, He: heliophilic).

\begin{tabular}{|c|c|c|c|c|c|c|c|c|c|c|}
\hline \multirow[t]{2}{*}{ Species } & \multicolumn{2}{|c|}{$\begin{array}{l}\text { Tolerance } \\
\text { to habitats }\end{array}$} & \multicolumn{3}{|c|}{$\begin{array}{l}\text { Requirement } \\
\text { of humidity }\end{array}$} & \multicolumn{5}{|c|}{$\begin{array}{l}\text { Preference } \\
\text { of habitats }\end{array}$} \\
\hline & $S$ & $\mathrm{E}$ & $x$ & M & $\mathrm{Hi}$ & $\mathrm{D}$ & $\mathrm{Hr}$ & $\mathrm{Ep}$ & Ps & $\mathrm{He}$ \\
\hline Calodromius spilotus & + & & & & + & + & & & & \\
\hline Cymindis angularis & & + & + & & & & & + & + & + \\
\hline Cymindis humeralis & & + & + & & & & & + & + & + \\
\hline Cymindis macularis & + & & + & & & & & + & + & + \\
\hline Cymindis vaporariorum & & + & + & & & & & + & + & \\
\hline Demetrias atricapillus & & + & & + & & & + & & & \\
\hline Demetrias imperialis & + & & & & + & & + & & & \\
\hline Demetrias monostigma & & + & & & + & & + & & & \\
\hline Dromius agilis & + & & & + & & + & & & & \\
\hline Dromius fenestratus & + & & & + & & + & & & & \\
\hline Dromius quadraticollis & + & & & + & & + & & & & \\
\hline Dromius quadrimaculatus & + & & & + & & + & & & & \\
\hline Dromius schneideri & + & & & + & & + & & & & \\
\hline Lebia chlorocephalus & & + & & + & & & + & & & \\
\hline Lebia cyanocephala & + & & + & & & & + & & & \\
\hline Lebia cruxminor & & + & & + & & & + & & & \\
\hline Lionychus quadrillum & + & & + & & & & & + & & + \\
\hline Microlestes maurus & & + & + & & & & & + & + & + \\
\hline Microlestes minutulus & & + & + & & & & & + & + & + \\
\hline Odacantha melanura & + & & & & + & & + & & & \\
\hline Paradromius linearis & & + & + & & & & & + & + & \\
\hline Paradromius longiceps & + & & & & + & & + & & & \\
\hline Philorhizus sigma & & + & & & + & & & + & & \\
\hline Syntomus foveatus & & + & + & & & & & + & + & + \\
\hline Syntomus truncatellus & & + & + & & & & & + & + & + \\
\hline Numb & 11 & 14 & 11 & 9 & 5 & 6 & 8 & 11 & 5 & 7 \\
\hline Proportion (\%) & 44 & 56 & 44 & 36 & 20 & 24 & 32 & 44 & 20 & 28 \\
\hline
\end{tabular}

cies, such as Demetrias monostigma, Calodromius spilotus, Dromius agilis, D. quadraticolis, D. schneideri, D. quadrimaculatus, Microlestes minutulus, Paradromius linearis, Philorhizus sigma, Lebia chlorocephala, L. cruxminor, Syntomus foveatus, $S$. truncatellus, and Odacantha melanura are widespread in the territory of the republic and should not be considered as rare. Nevertheless distributions of species such as Cymindis angularis, C. macularis, C. vaporariorum, Demetria imperialis, D. atricapillus, Dromius fenestratus, Microlestes maurus, Paradromius longiceps, and Lionychus quadrillum are fragmentary, localized in separate areas. These species are important in biodiversity conservation.

\subsection{Ecological characteristics of Lithuanian species of Lebiinae}

As it was mentioned in an introductory part of this paper, Lebiinae are distinguished by high diversity of ecological characteristics and biology. Therefore to describe their general ecological characteristics is not easy. In regard to key features of their lifestyle, we have identified the ten most important ecological criteria characterizing them (Table 2). According to habitat tolerance, Lebiinae ground beetles can be divided into two basic groups: stenobionts and eurybionts. Of the species, $11(44 \%)$ were attributed to the first group and $14(56 \%)$ to the second one. The majority of the stenobiontic species are closely re- 
lated to trees. Some ground beetles belonging to the genus Dromius prefer certain tree species over others; for example Dromius schneideri and Calodromius spilotus live almost exclusively on coniferous trees and $D$. quadrimaculatus dwell only on deciduous trees. Other species of this genus tolerate a variety of tree species, such are $D$. agilis, $D$. fenestratus and $D$. quadraticollis. The other quite large group of stenobiontic species is related to reeds: Odacantha spp. and some species of Demetrias and Paradromius genera. Nevertheless, some species belonging to these genera are characterized by a greater habitat tolerance or association even to two different habitats. In this aspect, an interesting species, Demetrias monostigma, can be mentioned. This is a typical species found in reeds, but it can be also found in dry, sandy habitats with Poaceae family plants. For this reason the species was attributed as a eurybiont.

According to water demand Lebiinae fauna of Lithuania is dominated by xerophiles (12 species $-48 \%$, Table 2 ), although some species of this group have the feature to tolerate higher moisture of habitats. For example, Cymindis vaporariorum tolerates moderate shade, temporary moist forest edges, Microlestes minutulus tolerates moderately dry, loamy soils, while Syntomus truncatellus is commonly found in open, moderately dry and also in semi moist habitats. Some xerophilic species clearly demonstrate their preference to sandy soils and are heliophilic. All Lebiinae species that are dendrobionts we considered as mezophilic, but indeed they are more related with inhabited tree species than depended on humidity. Altogether there are eight mezophilic Lebiinae species in Lithuania. Only five species were considered as higrophilic species, and they are mainly associated with reeds (except Philorhizus sigma that shows indifference to vegetation).

According to life styles, Lebiinae fauna of Lithuania was divided into the three groups: dendrobionts, herbobionts and epigeous species (Table 2). Most of them are attributed to the epigeous group (11 species). Eight species are considered herbobionts and six species dendrobionts.
Acknowledgements. We would like to thank Dr. Povilas Ivinskis, Dr. Vidmantas Monsevičius, Dr. Bronius Šablevičius, Mr. Romas Ferenca and Mr. Aleksandras Meržijevskis for loaning their material for examination. We are grateful to Dr. Alexander V. Anichtchenko (Institute of Systematic Biology, Latvia) and Dr. Oleg R. Aleksandrowicz (Academia Pomorska w Slupsku, Poland) for disscusion, assistance in beetle identification and useful comments on this study. Many thanks to Ms. Giedre Pakeltyte (Kaunas T. Ivanauskas Zoological Museum, Lithuania), Mr. Jim Owens (United States of America) and Mr. Kieran Flood (Ireland) for language improvements of the manuscript.

\section{References}

Anichtchenko, A. V. 2007-2012: Taxonomy of Lebiini. - In: Anichtchenko, A. V. (ed.), Carabidae of the World. [www document]. URL http://www.carabidae.org. (Site visited on 01 February, 2013).

Aleksandrowicz, O. R. 1991: (Ground beetles (Coleoptera, Carabidae) of the Belarus fauna). - In: Lopatin, I. K. \& Khotko, E. I. (eds.), Fauna i ekologia zhestkokrylykh Belorussii: 37-78. Nawuka i technika, Minsk. 263 pp. [In Russian.]

Aleksandrowicz, O. R. 1993: (The ecologo-faunistic review of ground beetles of western part of forest zone of Russian plain). - Veras, Minsk. 82 pp. [In Russian.]

Aleksandrowicz, O. R. \& Maksimenkov, M. V. 1994: (Annotated check list of ground beetles (Coleoptera: Carabidae) in Nalibojskaja puscha forest). - Akademija Nauk Belarusii, Minsk. 28 pp. [In Russian.]

Alekseev, V. I. 2008: Check-list of the ground beetles (Coleoptera, Carabidae) of Kaliningrad region. - Acta Biologica Universitatis Dauygavpiliensis 8(2): 153 191.

Anderson, R. 2006: Lebia cruxminor - a ground beetle. Northern Ireland Priority species. Biodiversity Unit, Northern Ireland Environment Agency [www document]. URL http:/www.habitas.org.uk/priority/species.asp?item $=7460$. (Site visited on 30 October, 2012).

Barševskis, A. 1987: Dažas ziņas par Latvijas dienvidaustrumi daļas skrejvaboḷu faunu (Some data on the fauna of ground beetles from the south-eastern pert of Latvia). - Latvijas Entomologs 30: 8-14. [In Latvian.]

Barševskis, A. 2001: New and rare species of beetles (Insecta: Coleoptera) in the Baltic countries and Byelorus. - Baltic Journal of Coleopterology 1(1-2): 3-18.

Barševskis, A. 2003: Latvijas skrejvaboles (Coleoptera: Carabidae: Trachypachidae \& Rhysodidae). — Baltic Institute of Coleopterolgy, Daugavpils 264 pp. [In Latvian.]

Bercio, H. \& Folwaczny, B. 1979: Verzeichnis der Käfer Preussens. - Parzeller \& Co, Fulda. 369 pp.

Bukejs, A. \& Telnov, D. 2010: On Latvian Chrysomelinae (Coleoptera: Chrysomelidae): 4. Genus Chrysolina 
Motschulsky, 1860. — Acta Zoologica Lituanica 20(2): 133-150.

Bukejs, A., Ferenca, R. \& Tamutis V. 2011: New and insufficiently known leaf-beetles species (Coleoptera: Chrysomelidae) of Lithuanian fauna. - Acta Zoologica Lituanica 21(2): 107-112.

Burakowski, B., Mroczkowski, M. \& Stefańska, J. 1974: Katalog fauny Polski, Tom. 3: Chrz szcze - Coleoptera.Biegaczowate - Carabidae. — Państwowe Wydawnictwo Naukowe Warzawa. 430 pp. [In Polish.]

Cibulskis, R. 1997: Materiali par Latvijas faunai jaunām un retām vaboḷu (Coleoptera) sugām. - Acta Coleopterologica Latvica 1(2): 72-76. [In Latvian.]

Clayhill, T. 1984: Lionychus quadrillum (Duftshmid) (Carabidae) found in Finland. - Notulae Entomologicae 64(4): 199.

Desender, K. R. C. 1996: Diversity and dynamics of coastal dunes carabids. - Annales Zoologini Fennici 33: 65-75.

Dvilevičius, R., Monsevičius, V. \& Švitra, G.1988: (Fauna and Biotopic Distribution of Carabidae and Staphylinidae (Coleoptera) in the Dukstyna Entomological Reserve of the Lithuanian SSR). - Acta Entomologica Lituanica 9: 27-36. [In Russian.]

Felix, R. \& van Wielink, P. 2011: Calodromius bifasciatus and other Carabidae on 26 oak-trunks in a nature reserve in the Netherlands. - In: Kotzc, D. J., Assmann, T., Noordijk, J., Turin, H. \& Vermeulen, R. (eds.), Carabid beetles as bioindicators: Biogeographical, Ecological and Environmental Studies: 533-544. Pensoft Publishers, Sofia, Moscow, ZooKeys 100. 573 pp.

Ferenca, R. 1988: (9 species of beetles new to the Lithuanian SSR, found in 1981-1985). — In: Jonaitis, V. (ed.), New and Rare for the Lithuanian SSR Insect Species. Records and Descriptions of 1987: 22-26. Institute of Zoology and Parasitology of the Academy of Sciences of the Lithuanian SSR, Lithuanian Entomological Society, Vilnius. 108 pp. [In Russian.]

Ferenca, R., Ivinskis, P. \& Meržijevskis, A. 2002: New and rare Coleoptera species in Lithuania. - Ekologija 3: $25-31$.

Ferenca, R. 2004: New and rare for Lithuania beetles (Coleoptera) species registered in 1978-2004. — New and rare for Lithuania insect species, Records and descriptions 16: 11-22.

Ferenca, R. 2006: A. Palionio vabalų rinkiniai. - In: Ivinskis, P. \& Rimšaite, J. (eds.), Entomologas Alfonsas Palionis (1905-1957): 162-216. Vilniaus Universiteto Ekologijos Institutas, Vilnius. 295 pp. [In Lithuanian.]

Ferenca, R., Ivinskis, P. \& Tamutis, V. 2006: New and rare for Lithuania species of beetles (Coleoptera). - New and rare for Lithuania insect species, Records and descriptions 17: 11-21.

Freude, H. 1976: Familie Carabidae. Unterfamilies Collurinae (Odacanthinae) und Lebiinae. - In: Freude, H., Harde, K. W. \& Lohse, G. A. (eds.), Die Käfer Mitteleuropas, 2 Band. Adephaga 1: 260-285. Goecke \& Evers, Krefeld. 302 pp.

Gorodkov, K. B. 1984: (Ranges types of insects of tundra and forest zones of European part of USSR). - In: Gorodkov, K. B. (ed.), (The Ranges of insects of of European part of USSR), Issue 5: 3-20. Nauka, Leningrad. 150 pp. [In Russian.]

Haberman, H. 1968: Eesti jooksiklased (Coleoptera, Carabidae). — Kirjastus "Valgus", Tallinn, 598 pp. [In Estonian.]

Hurka, K. 1996: Carabidae of the Czech and Slovak Republics. Illustrated key. - Kabourek. Zlin. 565 pp.

Inokaitis, V. 2004: Naujos ir retos Lietuvos entomofaunos vabalų (Coleoptera) rūšys, aptiktos 2000-2003 metais (New and rare foer the Lithuanian fauna Coleoptera species found in 2000-2003) — New and rare for Lithuania insect species, Records and description 16:710. [In Lithuanian.]

Ivinskis, P., Pakalniškis, S. \& Ferenca, R. 1998: Kiaulyčios pelkès entomofauna [abstract]. Biologinès ịvairovès tyrimai ir aplinkosauginis švietimas regionuose, 18 - 19 December, 1998, Marijampolè, Lithuania, 67 - 68. [In Lithuanian.]

Kabak, I. 2003: Carabidae: Lebiinae, Lebiini. — In: Löbl, I. \& Smetana, A. (eds.), Catalogue of Palaearctic Coleoptera, Vol. 1: Archostemata-Myxophaga - Adephaga: 408-439. Apollo Books, Stenstrup. 819 pp.

Karjalainen, S., Salokannel, J. \& Albrecht A. 2010: Atlas of the beetles of Finland. Interactive database. [www document]. URL http://www.luomus.fi/elaintiede/kovakuoriaiset/Catlas1.html. (Site visited on 01 February, 2013).

Koch K. 1989: Die Käfer Mitteleuropas. Ökologie Bd. 1. — Goecke \& Evers Verlag, Krefeld. 440 pp.

Lindroth, C. H 1954: Die larve von Lebia chlorocephala Hoffm. (Coleoptera: Carabidae). — Opuscula entomologica 19: 29-32.

Lindroth, C. H. 1992: Ground beetles (Carabidae) of Fennoscandia. A zoogeographic study. Part I. Specific knowledge regarding the species. - Amerind Publishing Co. Pvt. Ltd., New Delhi. 630 pp.

Ljunberg, H., Andrén, B., Ehnström B., Jansson, N., Jonsell, M., Lindelöw,.., Lundberg, S., Malmqvist, A., Nilsson, S. G., Pettersson, R. B., Ramqvist, T., Wanntorp H.-E. \& Wikars, L.-O. 2010: Skalbaggar - Beetles (Coleoptera). - In: Gärdenfors, U. (ed.), 2010. Rödlistade arter i Sverige 2010 - The 2010 redlist of Swedish species: 411-451. ArtDatabanken SLU, Uppsala. 589 pp.

Lönnberg, L. \& Jonsell, M. 2012: Sand pits as habitats for beetles (Coleoptera): does area affect species number and composition? - Biodiversity Conservation 21: 853-874.

Lopatin, I. K. \& Nesterova, O. L. 2005: (Insecta of Belarus: Leaf-beetles (Coleoptera, Chrysomelidae). Tehnoprint, Minsk. 294 pp. [In Russian.]

Lövei, G. L., Toft, S. \& Axelsen, J. A. 2005: Composition and diversity of spring-active carabid beetle assemblages in relation to soil management in organic wheat fields in Denmark. - In: Lövei, G. L. \& Toft, S. (eds.), European Carabodology 2003: Proceedings of the 11th European Carabidologist Meeting Århus (Denmark), July 2003. DIAS Report, 114 ed.: 173-182. 
Ministeriet for Fųdevarer, Landbrug og Fiskeri, Danmarks Jordbrugs Forskning, Forskningscenter Foulum, Markbrug. 401 pp.

Mazurowa, G. \& Mazur, E. 1939: Sprawozdanie z wycieczki koleopterologicznej do wojawództwa Wileńskiego v lipcu 1937. Przyczynek do znajomości fauny pón ${ }^{3}$ ocnej Polski. — Sprawozdanie Komisji Fizjograficznej 72: 463 476. [In Polish.]

Miländer, G., Monsevičius, V. \& Soo, V. 1984: (26 species of Coleoptera new to the Lithuanian SSR, found in 1974-1983). — In: Jonaitis, V. (ed.) New and rare for the Lithuanian SSR insect species. Records and descriptions of 1984: 23-30. Institute of Zoology and Parasitology of the Academy of Sciences of the Lithuanian SSR, Lithuanian Entomological Society, Vilnius. 91 pp. [In Russian.]

Monsevičius, V. 1997: Vabalai (Coleoptera). — In: Lapele, M. (ed.), Lietuvos valstybinių rezervatų flora ir fauna: 68-101. Aplinkos apsaugos ministerija, Vilnius. 168 pp. [In Lithuanian.]

Ogijewicz, B. 1933: Przycznek do znajomości chrsz'szczy (Adephaga and Palpicornia) okolik Wilna i Trok. Prace Towarzystwa Przyjació ${ }^{3}$ Nauk w Wilnie 7: 1 48. [In Polish.]

Ostrauskas, H. \& Ferenca, R. 2010: Beetles (Coleoptera) caught in traps baited with pheromones for Dendroctonus rufipennis (Kirby) (Curculionidae: Scolytinae) in Lithuania. - Ekologija 56(1-2): 41- 46.

Pileckis, S. 1960: Indèlis i Lietuvos vabalų (Coleoptera) faunos pažinim ${ }^{1}$. — L Ľ̃̄A mokslo darbai 7(3):303336. [In Lithuanian.]

Pileckis, S. 1963: Naujos vabalų (Coleoptera) rūšys Lietuvos TSR. — LŽŨA mokslo darbai 10(19): 53-64. [In Lithuanian.]

Pileckis, S. 1976: Lietuvos vabalai. — Mokslas, Vilnius, Lithuania. 244 pp. [In Lithuanian.]

Pileckis, S. \& Monsevičius, V. 1982: (65 new and 3 very rare for the Lithuanian SSR Coleoptera species found in 1971-1980). - In: Jonaitis, V. (ed.), New and rare for the Lithuanian SSR insect species. Reports and accounts of 1981: 12-30. Institute of Zoology and Parasitology of the Academy of Sciences of the Lithuanian SSR, Lithuanian Entomological Society, Vilnius. 71 pp. [In Russian.]

Pileckis, S. \& Monsevičius, V. 1995: Lietuvos fauna. Vabalai (Coleoptera) 1d. - Mokslas, Vilnius. 304 pp. [In Lithuanian.)]

Radkevich, A. I. 1970: (The ground beetles (Carabidae) as entomophagous in the fields and forests of Belorussian Pozerie). - In: Radkevich, A. I. (ed.), Zhivotnyj mir Belorusskogo Poserija: 90-113. Vitebskij Gosudarstvennyj Pedagogicheskij institut imeni S. M. Kirova, Minsk, BGU. [In Russian.].

Sazhnev, A. S. \& Eskov, I. D. 2010: (Parasitism of ground beetles for example of Lebia genus (Coleoptera, Carabidae)). - In: Vorotnikova, I. L. (ed.), Specialisty APK novogo pokolenija: Materialy IV Vserosijskoj nauchno-prakticheskoi konferencii: 192-193. Federalnoe gosudarstvennoe obrazovatelnoe uchrezhdenija vysshego professionalnogo obrazovanija, Sara- tovskij gosudarstvennyj agrarnyj universitet, Saratov. 264 pp. [In Russian.]

Sharova, I. H. \& Grüntal, S. J. 1973: (Carabidae Beetles (Coleoptera, Carabidae) of the reservation "Žuvintas" and of the Curonian Spit). - Acta entomologica Lituanica 2: 63-73. [In Russian.]

Sciaky, R. 1991: Revisione dei Philorhizus della regione Palearctica con descrizione di quattro nuovi taxa. Memorie della Societa Entomologija Italiana 69: 53 78 .

Schürstedt, H., Rossbach, A. \& Assmann, T. 2000: Morphological differentiations of tarsal structures in ground beetles living in reedbed habitats (Coleoptera, Carabidae). - In Brandmayr, P., Lovei, G., Brandmayr, T. Z., Casale, A. \& Vigna Taglianti, A. (eds.), Natural History and Applied Ecology of Carabid Beatles, Proceedings of the IX European carabidologists' Meeting (26-31 July 1998, Camigliatello, Cozensa, Italy): 81-87. Pensoft Publishers, Sofia-Moscow. 304 pp.

Silfverberg, H. 1992: Enumeratio Coleopterorum Fennoscandiae, Daniae et Baltiae. - Helsingfors Entomologiska Bytesförening, Helsinki 95 pp.

Silfverberg, H. 2004: Enumeratio nova Coleopterorum Fennoscandiae, Daniae et Baltiae. - Sahlbergia 9: 1111.

Silfverberg, H. 2010: Enumeratio renovata Coleopterorum Fennoscandiae, Daniae et Baltiae. - Sahlbergia 16(2): 1-144.

Sundukov, Yu., N. 2004: (A preliminary review of ground beatle genus Microlestes Schmidt-Goebel, 1846 (Coleoptera, Carabidae, Lebiini) of East Asia.) - Euroasian Entomological Journal 3(2): 119-128. [In Russian.]

Spuris, Z. 1983: Latvijas kukainu katalogs. 4. Skrejvaboles (Carabidae). - Latvijas Entomologs 26: 5-67. [In Latvian.]

Šablevičius, B. 2003: New and rare for Lithuania beetle (Coleoptera) species. - New and rare for Lithuania insect species, Records and descriptions 15: 11-24.

Tamutis, V. 2000: Carabid beetles species diversity and abundance in some agrobiocenoses. - In: Metspalu, L. \& Mitt, S. (eds.), Development of environmentally friendly plant protection in the Baltic region, Proceedings of the International Conference 28-29 September, 2000: 200-204. Tartu University Press, Tartu. 248 pp.

Tamutis, V., Ferenca, R., Ivinskis, P., Rimšaitè, J. \& Meržijevskis, A. 2008: New data on Lithuanian beetle (Coleoptera) fauna. - New and rare for Lithuania insect species, Records and descriptions 20: 29-38.

Tamutis, V. \& Dapkus, D. 2008: Kamšos Botaninis-Zoologinis draustinis. - In: Vaivilavičius, G. (ed.), Vabzdžiai ir moliuskai Kauno ir Kaišiadorių rajonu saugomose teritorijose: 41-46. Lututè, Kaunas. 96 pp. [In Lithuanian.]

Tamutis, V., Tamute, B. \& Ferenca, R. 2011: A catalogue of Lithuanian beetles (Insecta, Colepoptera). - ZooKeys 121: 1- 494.

Telfer, M. G. \& Eversham, B. C. 1996: Ecology and con- 
servation of heathland carabidae in eastern England. - Annales Zoologini Fennici 33: 133-138.

Telnov, D., Gailis, J., Kalninš, M., Napolov, A., Piterans, U., Vilks, K. \& Whitehead, P. F. 2005: Contributions to the knowledge of Latvian Coleoptera. 4. - Latvijas entomologs 42: 18-47.

Tsinkevich V. A. \& Aleksandrowicz, O. R. 2002: (New and rare species of beetles (Insecta, Coleoptera) of the Belarus fauna. - Vestnik Belorusskogo Gosudarstvennogo universiteta, Serija 2. Himija, Biologija, Geografija 2: 30-32. [In Russian.]

Zajančkauskas, P. \& Pileckis, S. 1968: (Coleoptera of the Žuvintas Reserve). - In: Zajančkauskas, P. (ed.), (Žuvintas Reserve): 264-282. Mintis, Vilnius. 397 pp. [In Russian.]
Vigna Taglianti, A. 2012: Carabidae. - In: Audisio, P. (ed.), Fauna Europaea: Coleoptera. Version 2.5. [www document]. URL http://www.faunaeur.org/ (Site visited on 07 February, 2013).

Žiogas, A. \& Gedminas, A. 1994: Lietuvos brukninių pušynų entomofauna (The entomofauna of Lithuanian vaccinium Scots pine stands). - Acta Entomologica Lituanica 12: 49-63. [In Lithuanian.]

Žiogas, A. \& Zolubas, P. 2005: Rare and protected forest beetle species in the national parks of Lithuania. - In: Sklodowski, J., Huruk, S., Barševskis, A. \& Tarasiuk, S. (eds.), Protection of Coleoptera in the Baltic Sea Region: 37-46. Warsaw Agricultural University Press, Warsaw. 239 pp. 